\title{
Effects of salicylate on intrinsic membrane properties of rat inferior colliculus neurons
}

\author{
By
}

Hamidreza Edrissi

A thesis submitted to the faculty of Graduate Studies and Research in partial fulfillment of the requirements for the degree of

\author{
Master of Science \\ Specialization in Neuroscience
}

\author{
Institute of Neuroscience \\ Department of Psychology \\ Carleton University \\ Ottawa, Ontario \\ April, 2007 \\ (C) Hamidreza Edrissi 2007
}




$\begin{array}{ll}\begin{array}{l}\text { Library and } \\ \text { Archives Canada }\end{array} & \begin{array}{l}\text { Bibliothèque et } \\ \text { Archives Canada }\end{array} \\ \begin{array}{l}\text { Published Heritage } \\ \text { Branch }\end{array} & \begin{array}{l}\text { Direction du } \\ \text { Patrimoine de l'édition }\end{array} \\ \begin{array}{l}\text { 395 Wellington Street } \\ \text { Ottawa ON K1A ON4 } \\ \text { Canada }\end{array} & \begin{array}{l}\text { 395, rue Wellington } \\ \text { Ottawa ON K1A ON4 } \\ \text { Canada }\end{array}\end{array}$

Your file Votre référence ISBN: 978-0-494-27017-2 Our file Notre référence ISBN: 978-0-494-27017-2

NOTICE:

The author has granted a nonexclusive license allowing Library and Archives Canada to reproduce, publish, archive, preserve, conserve, communicate to the public by telecommunication or on the Internet, loan, distribute and sell theses worldwide, for commercial or noncommercial purposes, in microform, paper, electronic and/or any other formats.

The author retains copyright ownership and moral rights in this thesis. Neither the thesis nor substantial extracts from it may be printed or otherwise reproduced without the author's permission.
AVIS:

L'auteur a accordé une licence non exclusive permettant à la Bibliothèque et Archives Canada de reproduire, publier, archiver, sauvegarder, conserver, transmettre au public par télécommunication ou par l'Internet, prêter, distribuer et vendre des thèses partout dans le monde, à des fins commerciales ou autres, sur support microforme, papier, électronique et/ou autres formats.

L'auteur conserve la propriété du droit d'auteur et des droits moraux qui protège cette thèse. $\mathrm{Ni}$ la thèse ni des extraits substantiels de celle-ci ne doivent être imprimés ou autrement reproduits sans son autorisation.
In compliance with the Canadian

Privacy Act some supporting forms may have been removed from this thesis.

While these forms may be included in the document page count, their removal does not represent any loss of content from the thesis.
Conformément à la loi canadienne sur la protection de la vie privée, quelques formulaires secondaires ont été enlevés de cette thèse.

Bien que ces formulaires aient inclus dans la pagination, il n'y aura aucun contenu manquant. 


\begin{abstract}
Tinnitus is as an auditory perception without an external auditory source. The inferior colliculus (IC) is a major structure in the central auditory pathway. It is one of the main candidates to be studied because of its possible involvement in generation of tinnitus. Salicylate is a well-known substance to produce reversible tinnitus in humans and animals. However, the neural origins and mechanisms of salicylate-induced tinnitus have not yet been fully clarified. The purpose of this study was to investigate the effects of salicylate on intrinsic membrane properties of neurons in the central nucleus of IC (ICC). Whole-cell patch clamp recordings were made from ICC neurons in a rat brain slice, under current clamp mode. Application of salicylate did not have any effect on the passive membrane properties of the ICC neurons. Salicylate also showed no effect on action potential properties except for reduction of afterhyperpolarization amplitude. However, salicylate affected firing abilities of the ICC neurons, including the firing threshold and frequency. The results suggest that salicylate increases membrane excitability of ICC neurons, which may account for an increase in spontaneous activity of ICC neurons, thus contributing to the generation of tinnitus.
\end{abstract}




\section{Table of Contents}

1. Introduction 1

1.1 The inferior colliculus: afferent and efferent projections 1

1.2 Subdivisions of the IC 2

1.3 Central nucleus of the inferior colliculus 2

1.3.1 Neuronal organization of the IC 2

1.3.2 Functional role of the ICC 3

1.3.3 Intrinsic firing patterns of the ICC neurons 4

1.4 Tinnitus 4

1.4.1 Etiology of tinnitus 5

1.4.2 Neural mechanisms of tinnitus 6

1.5 Salicylate role in induction of tinnitus 9

1.5.1 Saicylate dosage in human and animal studies $\quad 10$

1.5.2 Mechanisms of salicylate induced tinnitus 11

1.6 Effects of salicylate on the spontaneous activity of the IC neurons 12

$\begin{array}{lll}1.7 & \text { Effects of salicylate on neurotransmitters } & 13\end{array}$

1.7.1 Salicylate effects on glutamatergic activities of the SG neurons 13

1.7.2 Salicylate effects on GABAergic activities of the IC neurons 14

1.7.3 Salicylate effects on serotonergic activities of the IC neurons 16

$\begin{array}{lll}1.8 & \text { Effects of salicylate on voltage-gated ion channels } & 18\end{array}$

1.8.1 Effects of salicylate on voltage- gated sodium channels 18

1.8.2 Effects of salicylate on voltage-gated potassium channels $\quad 19$

1.8.3 Effects of salicylate on voltage-gated calcium channels 22

2. Purpose of the study 24

3. Materials and methods $\quad 25$

$\begin{array}{ll}\text { 3.1 Brain slice preparation } & 25\end{array}$

3.2 Whole-cell patch clamp recording 26 
$\begin{array}{lll}3.3 & \text { Data analysis } & 29\end{array}$

$\begin{array}{lll}\text { 4. Results } & 31\end{array}$

4.1 Effects of salicylate on passive membrane properties 31

4.1.1 Resting membrane potential 31

4.1.2 Current-voltage relationship and input resistant $\quad 32$

4.1.3 Time constant $\quad 32$

4.2 Effects of salicylate on membrane excitability 32

4.2.1 Firing pattern $\quad 32$

$\begin{array}{lll}\text { 4.2.2 Firing abilities } & 34\end{array}$

4.2.2.1 Threshold of firing $\quad 34$

4.2.2.2 Firing frequency $\quad 35$

4.3 Effects of salicylate on action potential properties 39

4.3.1 Action potential 39

$\begin{array}{ll}\text { 4.3.2 Afterhyperpolarization } & 39\end{array}$

4.3.3 Hyperpolarization-activated current $\left(I_{h}\right) \quad 40$

4.3.4 Rebound after hyperpolarization $\quad 41$

Table $1 \quad 42$

Figure 1-14 43

$\begin{array}{lll}\text { 5. Discussion } & 71\end{array}$

$\begin{array}{lll}\text { 5.1 Salicylate and passive membrane properties } & 71\end{array}$

$\begin{array}{lll}5.2 & \text { Salicylate and membrane excitability } & 71\end{array}$

$\begin{array}{lll}5.2 .1 & \text { Firing pattern } & 71\end{array}$

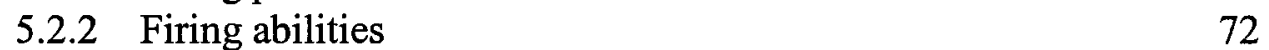

5.2.2.1 Threshold of firing $\quad 72$

$\begin{array}{ll}\text { 5.2.2.2 Firing frequency } & 73\end{array}$

$\begin{array}{lll}5.3 & \text { Salicylate and action potentials properties } & 77\end{array}$

$\begin{array}{lll}\text { 5.3.1 Action potential } & 77\end{array}$

$\begin{array}{lll}\text { 5.3.2 Afterhyperpolarization } & 77\end{array}$

5.3.3 Hyperpolarization-activated current $\left(\mathrm{I}_{\mathrm{h}}\right) \quad 78$

$\begin{array}{ll}\text { 5.3.4 Rebound after hyperpolarization } & 79\end{array}$

5.4 Effects of salicylate on ICC neurons and tinnitus $\quad 79$ 
$\begin{array}{ll}\text { 6. Conclusion } & 82\end{array}$

References 


\section{List of Abbreviations}

4-AP

5-HT

AI

AII

ACSF

AHP

AMPA

AP

CNS

DCN

GABA

GAD

HEPES

IC

ICC

ICD

ICX

$\mathrm{I}_{h}$

$\mathrm{I}_{\mathrm{K}(\mathrm{A})}$

$\mathrm{I}_{\mathrm{K}(\mathrm{DR})}$

$\mathrm{I}_{\mathrm{Na}}$

IV

NMDA

$\mathrm{R}_{\text {in }}$

RLF

RMP

SFR

$\mathrm{SG}$

$\tau$

TEA

TTX 4-aminopyridine

serotonin or 5-hydroxytryptamine

primary auditory cortex

secondary auditory cortex

artificial cerebrospinal fluid

after-hyperpolarization potential

$\alpha$-amino-3-hydroxy-5-methyl-4-isoxazolepropionic acid

action potential

central nervous system

dorsal cochlear nucleus

$\gamma$-aminobutyric acid

glutamic acid decarboxylase

N-2-hydroxyethylpiperazine-N'-2-ethanesulfonic acid inferior colliculus

central nucleus of the inferior colliculus

dorsal cortex of the inferior colliculus

external cortex of the inferior colliculus

Hyperpolarization-activated current

transient outward potassium current

delayed rectifier potassium current

sodium current

current-voltage

$\mathrm{N}$-methyl-D-aspartate

input resistance

rate level function

resting membrane potential

spontaneous firing rates

spiral ganglion

time constant

tetraethylammonium

tetrodotoxin 


\section{Introduction}

\subsection{The inferior colliculus: afferent and efferent projections}

The auditory system is composed of numerous nuclei that are connected by parallel and series pathways. The pathways from various neuronal groups in the auditory lower brainstem converge on a common destination, the inferior colliculus (IC). The inferior colliculus is the main auditory structure of the mesenceplaon, forming a clearly visible protuberance on its dorsal structure (Rouiller, 1997). It receives and integrates ascending afferent projections from almost all of the major auditory nuclei in the lower brain stem. It also receives the inputs from the opposite IC. Auditory information that has been encoded in specific temporal or spatial configurations in the lower brain stem nuclei is further analyzed, relayed, or transformed in the IC. The wealth of afferent inputs includes excitatory and inhibitory projections. The cochlear nucleus (CN), medial superior olive (MSO), and contralateral lateral superior olive (LSO) are sources for major excitatory inputs. The dorsal nucleus of the lateral lemniscus (DNLL), ipsilateral LSO, and ventral nucleus of the lateral lemniscus (VNLL) are sources for major inhibitory inputs. The IC also receives descending projections from the auditory cortex and medial geniculate body (MGB), and local inputs from neurons within the IC. In addition, the IC receives converging auditory, somatosensory, visual, and motor information (Wu, 2005). Thus, IC neurons are influenced by ascending, descending, and internal excitatory and inhibitory synaptic inputs while processing auditory and other signals. The IC provides the principal source of innervations to the medial geniculate body and indirectly to the auditory cortex. Therefore, the IC processes and integrates almost all ascending acoustic 
information from lower auditory centers, and then determines the form in which acoustic information is conveyed to higher regions in the forebrain.

\subsection{Subdivisions of the IC}

The internal structure of the IC is characterized by the distribution of different cell types, patterns of dendritic branching and the termination of afferent, intrinsic and commissural fibers. Several attempts have been made to subdivide the IC using different methods such as Golgi staining, Nissl staining, and immunocytochemistry with the hope that these subdivisions would correspond to functional zones. Using Nissl-stained sections, Golgi impregnations and myelin stains, Faye-Lund and Osen (1985) subdivided the rat IC into three main areas: the central nucleus (ICC), the laterally and rostrally placed external cortex (ICX), and the dorsal cortex (ICD). This classification scheme is the most basic and simple anatomical subdivision that is easily related to sensory neurophysiology. Each subdivision has unique afferent and efferent connections. The cell type and size, dendritic field shape and organization are also different in these three nuclei. Using histological techniques, the abrupt changes in soma or dendritic orientation are very helpful in determination of the nuclei and their boundaries.

\subsection{Central nucleus of the inferior colliculus}

\subsubsection{Neuronal organization of the ICC}

The central nucleus of the IC is the main division of the mammalian IC. It is characterized by the presence of fibrodendritic laminae distinguishable in Golgi materials, which is composed of disc-shaped neurons and the laminar plexus of afferent 
fibers (Oliver, 2005). Two major cell types have been defined by morphology in the ICC of the cat, disc-shaped neurons with dendritic arbors and stellate cells with radiating dendritic arbors that often transverse the laminae. Disc-shaped neurons are the most common cell type in the ICC. They have dendritic fields parallel to one another and contribute a distinct laminar appearance in Golgi stains. The thickness of the dendritic trees determines the width of the laminae which is about $70-150 \mu \mathrm{m}$. Stellate neurons, the second main cell type, represent less than one fourth of the total population. Their dendrites extend beyond the single fibrodendritic lamina into adjacent laminae. This laminar organization offers the structural basis for the tonotopic organization.

\subsubsection{Functional role of the ICC}

The ICC is the major auditory integrative center in the central auditory pathway and it is essential for normal hearing (Winer, 2005). The auditory information from many sources of the brainstem is collected and reorganized into well-ordered spatial and temporal patterns of excitation that can be understood in higher centers (Ehert, 1997). The frequency-band laminae containing thousand of neurons with a similar characteristic frequency represent the main feature of the ICC organization (Winer, 2005). Distinct laminae of the ICC are related to the corresponding sound frequencies. Considerable data have shown that the ICC is associated with the topographical representations of frequency, sharpness of the frequency tuning, and binaural processing (Aitkin, 1986).

In addition, it is well known that most natural sounds including the communication sounds contain frequency and amplitude modulations. These modulations generate a temporal structure, which is a major source of information for recognizing sounds. Considerable data have shown that the ICC neurons can extract the temporal 
auditory information like the periodicity of amplitude- or frequency-modulated sounds found in complex signals (Koch and Grothe, 1998, 2000). Therefore, the ICC is the vital integrative center in the central auditory system.

\subsubsection{Intrinsic firing pattern of the ICC neurons}

Different IC neurons respond selectively and specifically to intracellular injection of depolarizing and hyperpolarizing current (Li et al., 1998; Peruzzi et al., 2000; Wu et al., 2002). Depolarizing current injection activates voltage-sensitive ion channels and generates action potentials with different firing patterns. Based on firing patterns, the ICC neurons represent at least six physiological types to combinations of depolarizing and hyperpolarizing current injections, which include sustained-regular, rebound-regular, rebound-adapting, rebound-transient, onset and buildup-pause patterns (Sivaramakrishnan and Oliver, 2001). These firing patterns are correlated with different combinations of specific $\mathrm{K}^{+}$channels.

\subsection{Tinnitus}

Tinnitus is an auditory phantom sensation (ringing of the ears) experienced when no external sound is present. Most but not all cases are associated with hearing loss induced by noise exposure or aging (Eggermont and Roberts, 2004). Many (79\%) tinnitus sufferers report that tinnitus sounds like tones (including ringing, chirping), whereas $21 \%$ report it as noise (hissing steam, rushing water, ocean waves). The physiological correlate of perceived sound is a neural activity pattern in auditory cortex that differs from normal spontaneous activity. These changes can be an increased spontaneous firing rate, increased synchrony between the firings of neurons in cortex or changes in the firing 
patterns of small groups of neurons. Potentially, any change in firing rate, neural synchrony or burst firing pattern relative to normal spontaneous activity could be a neural correlate of tinnitus.

Plasticity of the adult mammalian nervous system has been demonstrated to occur in a number of sensory modalities and central neural structures in response to sensory deprivation. Cortical tonotopic organization is altered in both aging and following selective deafferentation (Willott et al., 1993). Altered receptor characteristics, neurotransmitter expression, and synaptic structural change provide possible mechanisms for central auditory plastic changes in order to improve auditory detection, frequency discrimination, binaural hearing and topographic representation. While plasticity may be an adaptive response to altered stimulus environments, plasticity may also result in abnormal sensory perception. Tinnitus may be one such pathological manifestation of auditory plasticity.

\subsubsection{Etiology of tinnitus}

In humans, the main identified tinnitus-producing agents are noise trauma (20\%), head and neck injury (9\%), ear problems and colds (7\%), ototoxic drugs $(2 \%)$ and Meniere's disease. The remaining $60 \%$ have unknown or various etiologies (Eggermont, 2003). Typically, the sensation is associated with a reversible cause such as listening to loud music, fever, use of aspirin or quinine, or transient perturbations of the middle ear, which cause reversible tinnitus and subsides over a period ranging from a few seconds to a few days (Eggermont and Robert, 2004). However, in 5-15\% of the general population, the tinnitus sensation is unremitting (Heller, 2003). Chronic tinnitus is more prevalent among seniors (12\% after age 60$)$ than in young adults (5\% in the $20-30$ age groups) but 
can occur at any age. In $1-3 \%$ of the general population, the tinnitus sensation is sufficiently loud to affect the quality of life, involving sleep disturbance, work impairment and psychiatric distress (Dobie, 2003). Most cases of chronic tinnitus are associated with hearing loss that is induced by noise exposure or it accompanies the aging process. The prevalence of tinnitus could be increasing as the senior population grows and as young people are increasingly exposed to industrial and recreational noise.

\subsubsection{Neuronal mechanisms of tinnitus}

The strong association between sensorineural hearing loss, cochlear injury, and tinnitus leads to early speculations that tinnitus is due to cochlear dysfunction (Zenner and Ernst, 1993). Tinnitus sensations associated with hearing loss are usually localized towards the affected ear(s). However, there is limited support for the assumption that tinnitus is the result of increased spontaneous activity in auditory nerve fibers. Evidence is found after high-dose application of salicylate (Evans et al.,1981), but low doses do not increase spontaneous firing rates (SFR) (Müller et al., 2003), even though tinnitus can be demonstrated behaviorally for low doses (Bauer et al 2000). Other ototoxic drugs that cause tinnitus, such as quinine and aminoglycosides, show a consistent decrease in the SFR of auditory nerve fibers (Mulheran, 1999). A similar decrease is reported after noiseinduced hearing loss (Liberman and Kiang, 1978). These results showing reduced SFR in auditory nerve fibers following noise exposure or ototoxicity point to a central cause of tinnitus, possibly related to changes in the balance of excitatory and inhibitory inputs conveyed to central auditory structures. For instance, tinnitus can be prevented if NMDA receptor blockers are infused into the cochlea before salicylate application (Guitton et al, 2003), and that prior administration of NMDA receptor blockers can limit hearing loss 
resulting from noise trauma (Duan et al., 2000). It seems that by reducing the extent of the hearing loss, probably by preventing the neurotoxic effects of excessive glutamate release at cochlear NMDA receptors, the tinnitus is also prevented. These findings are consistent with the view that the origin of tinnitus lies in an imbalance of firing patterns across the tonotopic array of auditory nerve fibers, but not with the view that tinnitus reflects increased spontaneous activity generated there.

Tinnitus sensations often persist even when input from the ear is removed by section of the auditory nerve (House and Brackman, 1981). Other studies also indicate that tinnitus can occur with normal cochlear function as well as after complete peripheral deafferentation (Eggermont, 1990; Jastreboff, 1990). Currently, most evidence based on SFR measurements points to changes in the central auditory system following dysfunction of the cochlear receptors as the source of tinnitus. Diminished output from the affected region causes reduced inhibition in central auditory structures (Milbrandt et al., 2000), leading to hyperexcitability of the central auditory system (Salvi et al., 2000). This reduced inhibition has been indirectly demonstrated, after low-dose salicylate application, by the increase in SFR of neurons in the central (ICC) and external (ICX) nuclei of the inferior colliculus (Jasterboff and Sasaki, 1986; Chen and Jastreboff, 1995) and in secondary auditory cortex (AII) (Eggermont and Kenmochi, 1998). In primary auditory cortex (AI), a low dose of salicylate did not produce changes in SFR (Ochi and Eggermont, 1996), whereas a high dose did. After cis-platin application (an ototoxic agent, which resulting in hearing loss, morphologic damage and biochemical changes in the cochlea), SFR was increased in the dorsal cochlear nucleus (DCN) of hamsters (Kaltenbach et al., 2002). Quinine application increased SFR in AII (Eggermont and 
Kenmochi, 1998) but not in AI (Ochi and Eggermont, 1997). Noise trauma increased SFR in DCN (Kaltenbach et al., 1998) and AI (Seki and Eggermont, 2003). These findings point to an increase in SFR in cortical and subcortical auditory structures following noise trauma and exposure to ototoxic drugs. Whether increases in SFR relate directly to the sensation of tinnitus is, however, unclear. An interesting aspect is that, although tinnitus is often experienced immediately after noise exposure, increases in SFR took a few hours to materialize in AI (Norena and Eggermont, 2003) and several days to appear in DCN (Kaltenbach et al., 2000). The temporal correlation of changes in SFR with the time course of tinnitus needs to be further investigated.

Two other possible correlates of tinnitus that have been investigated using animal models of hearing loss are burst firing and neural synchrony. Although burst firing increases after salicylate application in ICX (Chen and Jastreboff, 1995), in cortical neurons the amount of bursting observed after salicylate or quinine application (Ochi and Eggermont, 1997) or after noise trauma (Seki and Eggermont, 2003) does not change; transitory increases in $\mathrm{AI}$ after noise trauma return to baseline within a few hours (Norena and Eggermont, 2003). A second feature of spontaneous activity in AI, the synchronization of the firings of several neurons, is increased immediately after a noise trauma for neurons in the affected frequency region (Norena and Eggermont, 2003), and also after quinine application (Ochi and Eggermont, 1997). Synchrony in the affected frequency region also increases with time and relates to reorganization of the cortical tonotopic representation by noise trauma.

Evidence from human brain imaging studies confirms involvement of central structures in tinnitus and points to changes accompanying tinnitus not only in the inferior 
colliculus (Melcher et al., 2000) and auditory cortex but also in limbic structures associated with emotion (Lockwood et al., 2001). These structures are modulated by activation of auditory nuclei that are also innervated by non-auditory inputs (e.g. the trigeminal nerve), and can become activated as a consequence of removal of acoustic tumors or head and neck injury (Levin et al., 2003). Animal studies also suggest that metabolism after salicylate administration decreases in IC but increases in the auditory thalamus (medial geniculate body) and auditory cortex, as well as in the amygdala (Zhang et al., 2003). Therefore, despite the abundance of assumed locations of the generator of tinnitus, most current hypotheses agree that abnormal neural activity is interpreted and perceived as tinnitus in higher auditory centers.

\subsection{Salicylate role in induction of tinnitus}

After the development of aspirin, almost 150 years ago, it was noted that high doses of aspirin employed in the treatment of chronic inflammatory diseases was associated with auditory toxicity. Tinnitus, loss of absolute acoustic sensitivity and alterations of perceived sounds are the three auditory alterations described by human subjects after ingestion of large doses of salicylate (Müller et al., 2003). These symptoms develop over the initial days of treatment, may stabilize, fluctuate or decrease in intensity, and are reversible within a few days of cessation of treatment. They may also occur within hours of ingestion of an extremely large dose. Individual subjects vary notably as to their susceptibility to salicylate-induced auditory toxicity. Tinnitus may be the first subjective symptom, and is often described as a continuous high pitch sound of mild loudness (Cazals, 2000). The hearing loss is slight to moderate, bilaterally symmetrical 
and affects all frequencies with often predominance at the high frequencies. Alterations of perceived sounds include broadening of frequency filtering, alterations in temporal detection, deterioration of speech understanding and hypersensitivity to noise.

\subsubsection{Salicylate dosage in human and animal studies}

Salicylate has often been used to study tinnitus in animal models. In humans (Day et al., 1989; Boettcher and Salvi, 1991), high dosages of salicylate induce reversible tinnitus. This has also been shown to occur in rats (Jastreboff, 1990; Jastreboff and Sasaki, 1994). Salicylate causes elevated central auditory activity (e.g., Jastreboff and Sasaki, 1986; Chen and Jastreboff, 1995; Wallhausser-Franke et al., 1996; Ochi and Eggermont, 1996) and this increased activity has been suggested to be a neural correlate of tinnitus. It is unclear if this elevated central activity is generated in the periphery due to enhanced activity in the auditory nerve, because of conflicting results between studies on auditory nerve activity after salicylate application. In the cat, an increase in auditory nerve spontaneous activity was observed after $400 \mathrm{mg} / \mathrm{kg}$ salicylic acid (Evans and Borewe, 1982). In both the cat and the guinea pig a dose dependent effect was observed, $400 \mathrm{mg} / \mathrm{kg}$ salicylic acid resulted in an increased spontaneous activity, and $200 \mathrm{mg} / \mathrm{kg}$ in unchanged spontaneous activity (Stypulkowski, 1990). It has been shown that in the gerbil $200 \mathrm{mg} / \mathrm{kg}$ salicylic acid results in increased activity in the central auditory system (Wallhausser-Franke et al., 1996; Wallhausser-Franke, 1997). In humans, a dosage of $1.95 \mathrm{~g} /$ day per subject for 7 days is sufficient to induce tinnitus (Day et al., 1989); this corresponds to $28 \mathrm{mg} / \mathrm{kg} /$ day for a $70 \mathrm{~kg}$ Subject. In addition, the behavioral model in rats (Jastreboff and Sasaki, 1994) shows that a dosage of $200 \mathrm{mg} / \mathrm{kg}$ and below is sufficient to induce tinnitus or more carefully interpreted, as a change in behavior. 
Jastreboff et al. (1986) investigated the time course of salicylate concentrations in serum and cerebrospinal fluid in rats over 8 hours after intraperitoneal injection of sodium salicylate at $460 \mathrm{mg} / \mathrm{kg}$ body weight. Salicylate concentrations in serum reached a peak of about $700 \mathrm{mg} / \mathrm{l}$ (equivalent to $4.37 \mathrm{mM}$ ) within $1.5 \mathrm{~h}$ and slowly decreased to $500 \mathrm{mg} / \mathrm{l}$ (equivalent to $3.12 \mathrm{mM}$ ) over the next $6 \mathrm{~h}$. Correspondingly, salicylate concentration in cerebrospinal fluid rose to approximately $200-300 \mathrm{mg} / 1$ (equivalent to 1.25-1.87 mM) within $1 \mathrm{~h}$ and remained at this level for the next $3 \mathrm{~h}$, then decreased slowly to $150-200 \mathrm{mg} / 1$ (equivalent to $0.94-1.25 \mathrm{mM}$ ) in the next $4 \mathrm{~h}$. Study of shortterm, acute, induced phantom auditory sensations in rats showed that tinnitus was induced by the administration of sodium salicylate $(350 \mathrm{mg} / \mathrm{kg}$ body weight) given $3 \mathrm{~h}$ before testing (Lukas-Rüttiger et al., 2003). Thus, when tinnitus develops in rats treated with salicylate, its concentration in their cerebrospinal fluid is about $1 \mathrm{mM}$.

\subsubsection{Mechanisms of salicylate induced tinnitus}

The mechanisms of salicylate-induced tinnitus generation in the central auditory pathway have not yet been fully clarified. It has been shown that sodium salicylate reduces markedly the cochlear blood flow by vasoconstriction (Didier et al., 1993), and activates cochlear NMDA receptors (Guitton et al., 2003). Local application of salicylate to cochlear outer hair cells impairs their electromotile responses and leads to subsequent functional and structural changes, i.e. a reduction of electromotile length changes (Shehata et al., 1991) and lateral wall stiffness (Lue and Brownell, 1999). However, in addition to these reported peripheral (cochlear) effects some studies investigated the consequences of systemic salicylate application on central auditory structures. It was demonstrated that salicylate could reduce the energy consumption (Wallhausser-Franke 
et al., 1996) and c-fos expression (Wallhausser-Franke, 1997; Wallhausser-Franke et al., 2003) of the ascending auditory pathway up to the inferior colliculus and increase the GABAergic activity in the inferior colliculus (Bauer et al., 2000). The main common feature of systemic salicylate application is the modulation of neuronal spontaneous activity of the auditory nerve (Evans et al., 1981; Evans and Borerwe, 1982; Müller et al., 2003), the inferior colliculus (Jastreboff and Sasaki, 1986; Chen and Jastreboff, 1995; Manabe et al., 1997) or the auditory cortex (Ochi and Eggermont, 1996; Kenmochi and Eggermont, 1997; Eggermont and Kenmochi, 1998). However, because the activity of the auditory nerve decreased (in fibers with a best frequency below $5 \mathrm{kHz}$ ) or remained unchanged (in fibers with a best frequency above $5 \mathrm{kHz}$ ) after systemic salicylate intoxication, the elevated activity in the central auditory system cannot be caused by cochlear nerve hyperactivity (Müller et al., 2003). It is unclear yet to what extent peripheral or central salicylate effects contribute to the generation of tinnitus.

\subsection{Effects of salicylate on spontaneous activity of the IC neurons}

Considering the fact that the inferior colliculus (IC) has been described as a primary candidate structure for tinnitus generation (Manabe et al., 1997; Bauer et al., 2000), Basta and Ernst (2004) investigated the direct effect of salicylate application on the spontaneous activity of mouse inferior colliculus neurons in brain slices by extracellular recording. Out of 92 neurons, $87 \%$ responded statistically significantly to sodium salicylate by changing their firing rates $(70 \%$ increased and $17 \%$ decreased their firing rates, respectively and the rest remained unchanged). Therefore, salicylate superfusion induced a general increase of electrophysiological activity within the inferior 
colliculus, which was similar to those obtained during systemic application of salicylate. However, a recent study on the effect of acute salicylate treatment (injection of 200-300 $\mathrm{mg} / \mathrm{kg}$, i.p.) in mice (Ma et al., 2006), demonstrated a significant decrease of spontaneous rates in the inferior colliculus. In this study, salicylate elevated neural thresholds, broadened excitatory receptive fields, and suppressed spontaneous activity in the ICC of treated mice. Therefore, salicylate-induced changes in spontaneous activity remain controversial and differ considerably between studies. These results suggest that a generalized hyperactivity model may be inappropriate for the functionally diverse principal cells of the inferior colliculus.

\subsection{Effects of salicylate on neurotransmitters}

The characterization of neurotransmitter changes induced by salicylate in auditory neurons may provide insight into the action of salicylate on the auditory system and through this, provide a better understanding of neurological mechanism of salicylateinduced tinnitus.

\subsubsection{Effects of salicylate on glutamatergic activities of spiral ganglion neurons}

Peng et al., (2003), studied the effect of aspirin on the cochlear neurotransmission, and its interactions with major types of membrane channels and receptors regulating the excitability of cultured type I spiral ganglion (SG) neurons. Results showed that aspirin had little effect on voltage-gated sodium and potassium currents of SG neurons. In contrast, it selectively potentiated the N-methyl-D-aspartate (NMDA) subtype of the glutamate responses in SG neurons while showing little effect on the AMPA and kainate types of glutamate responses. The aspirin-induced current in the presence of NMDA 
increased in a dose-dependent manner with a half maximal concentration of $2.2 \mathrm{mM}$, and it was blocked by NMDA receptor antagonist 2-amino-5-phosphonopentanoic acid or $\mathrm{Mg}^{2+}$. These in vitro results suggested that aspirin could interfere with the glutamatergic neurotransmission in the cochlea by selectively amplifying NMDA-mediated responses.

The NMDA receptor is a subtype in the glutamate receptor family, whose activation is crucial in the induction of long-term potentiation that is believed to underlie learning and memory. Since strong evidence indicates that glutamate or a structurally related molecule is the principal afferent transmitter of cochlear hair cells, the salicylateinduced potentiation of NMDA responses in SG neurons suggests that the drug may activate some intracellular mechanisms resulting in a long-term modulation of neurotransmission between hair cells and spiral ganglion neurons. The signal from hair cells may be abnormally amplified, ultimately resulting in a tinnitus sensation.

\subsubsection{Effects of salicylate on GABAergic activities of IC neurons}

Another neurotransmitter of concern is GABA, which a number of studies have addressed the hypothesis that a change in GABA-mediated inhibition may result in tinnitus. For example, Brummett (1995) has proposed that tinnitus may result from a loss of GABA-mediated inhibition within the IC and a consequent increase in neural activity from the IC to the auditory cortex. Bauer et al. (2000) studied the effects of chronic salicylate on GABA system of the IC of the rats. The subjects were chronically treated with oral salicylate. One group was behaviorally tested and salicylate-induced tinnitus was detected using a conditioned suppression behavioral model. In the rats, demonstrating behavioral evidence of tinnitus there was a significant increase in the level of $\mathrm{GAD}_{65}$ protein in the IC. 
GAD (glutamic acid decarboxylase) is the rate-limiting enzyme in the formation of GABA and occurs as two isoforms, $\mathrm{GAD}_{65}$ and $\mathrm{GAD}_{67} . \mathrm{GAD}_{65}$ is considered a reservoir for local synaptic requirements since it is stored in axon terminals in predominantly inactive (apoenzyme) form (Kaufman et al., 1991). Houser and Esclapez (1996) have proposed that increased demands for GABA synthesis would potentially result in an increase in the $\mathrm{GAD}_{65}$ reservoir pool. The upregulation of $\mathrm{GAD}$ expression may occur as a compensatory inhibitory response to an increase in central auditory traffic that results from chronic salicylate exposure. Recovery from stimulation resulted in normalization of GAD levels. Chronic salicylate exposure may result in continuous stimulation of the auditory pathway resulting in an increase in $\mathrm{GAD}_{65}$ to supplement the availability of terminal GABA.

In Bauer's study, increased levels of $\mathrm{GAD}_{65}$ were confined to IC and were not present in other brain structures. This suggests that the altered inhibitory activity was a specific effect within the auditory system from chronic exposure to salicylate. Because GAD levels were not altered in non-auditory structures, chronic systemic administration of salicylate does not appear to exert a direct universal effect on GABA systems throughout the central nervous system. Therefore, the salicylate-induced increase in IC GAD either may occur because of a direct unique effect of salicylate on the auditory GABAergic system, or because of a GABAergic response to increased neural activity within the cochlea or eighth nerve induced by chronic salicylate.

Furthermore, $\mathrm{GABA}_{\mathrm{A}}$ receptor binding studies suggested that there was an increase in receptor affinity and a decrease in $\mathrm{GABA}_{\mathrm{A}}$ binding sites in subjects chronically treated with salicylate. The decrease in $\mathrm{GABA}_{\mathrm{A}}$ receptor binding sites may be 
a manifestation of tinnitus-related changes in GABA neurotransmission or input activity to the IC. Furthermore, the salicylate-induced increase in muscimol affinity to the $\mathrm{GABA}_{\mathrm{A}}$ receptor is suggestive of a compensatory change in $\mathrm{GABA}_{\mathrm{A}}$ receptor number and/or subunit composition.

The results of the Bauer (2000) study indicate that chronic exposure to salicylate induces receptor changes and alters expression of GAD within the auditory brainstem. These changes may represent aberrant plasticity clinically experienced as tinnitus.

\subsubsection{Effects of salicylate on serotonergic activities of IC neurons}

The effects of salicylate on serotonergic activities in rat IC were studied by Liu et al. (2003), who monitored these effects by microdialysis in salicylate-induced tinnitus animals. In this study, Wistar rats were treated with salicylate $(350 \mathrm{mg} / \mathrm{kg}$ i.p.), which produced significant increase in the IC glucose, lactate and serotonin levels.

Since extracellular glucose and lactate levels have been advocated for reflecting local neuronal activity, monitoring their levels in the extracellular fluid with microdialysis provides a powerful method for studying local neuronal activity in awake, freely moving animals. This study indicated that acute salicylate exposure may increase neuronal activity, regional cerebral blood fluid, and hence the glucose supply from blood. The increase in anaerobic glucose consumption during physiological activity has been well established during the last decade. Increases in cerebral lactate levels, which were previously considered to occur because of neuronal distress, are now regarded as a simple consequence of brain activation.

Salicylate also significantly increased the 5-HT level. Serotonin transmission forms a major modulatory network within sensory systems. This network influences 
various information-processing mechanisms and, in particular, filtering of auditory information. It has been shown in rats that 5-HT activation in sensory neurons increases with age (Cransac et al., 1996), and it may compensate for dysfunction of sensory input and processing. It therefore seems likely that the perception of tinnitus could be linked to a dysfunction of 5-HT at one or more levels in the central nervous system. Some data indicate that the primary cause of central tinnitus is related to 5-HT functions. Much of these understandings of 5-HT have come from immunocytochemical and biochemical studies. In Liu study (2003), dialysate 5-HT levels in IC significantly increased 2-3 h after salicylate application, while saline did not produce any clear changes, which suggests that the increased 5-HT levels in IC may be involved in tinnitus generation. Similar changes have been described in studies on insomnia. The release of 5-HT has been found to increase during waking and decrease during sleeping. This may explain why tinnitus is often accompanied by insomnia and is commonly reported to be at its worst on waking.

Working on changes in blood serotonin in patients with tinnitus and other vestibular disturbances, Sachanska (1999) found that patients with tinnitus had serotonin blood values that significantly exceed the serotonin levels in control subjects. Additionally, compared with the changes of glucose and lactate levels in IC, the increases of 5-HT levels were slower and more prolonged, which may indicate that 5-HT plays a role in the regulation of the behavioral response rather than its initiation, and may indicate that there are other neurotransmitters involved in these changes of neuronal activities. 


\subsection{Effects of salicylate on voltage-gated ion channels}

Electrical and chemical signaling in the nervous system is of fundamental importance to the integration of external and internal stimuli. Proper signaling depends on a finely tuned series of ion-channel-mediated events mediating electrical activity and transduction at synapses. Slight changes in ion channel properties will cause severe changes in physiological functions of neurons.

\subsubsection{Effects of salicylate on voltage-gated sodium channels}

Voltage-gated sodium channels mediate the very rapid rising phase and initial component of the falling phase of action potentials in the excitable cells. Voltage-gated sodium channels also play a critical role in action potential propagation as well as transduction of electrical currents to transmitter release from the nerve terminal. Since $\mathrm{Na}^{+}$is the primary inward charge carrier for action potentials in the IC neurons, changes in sodium channel properties or density might be responsible for some of the changes in neural excitability with salicylate use.

To investigate the effects of sodium salicylate, on voltage-gated sodium channels, Liu and Li (2004) studied freshly dissociated inferior colliculus neurons of rats by the whole-cell voltage clamp method. The results indicated that salicylate blocked sodium channels and decreased the amplitudes of $\mathrm{I}_{\mathrm{Na}}$ in a concentration-dependent manner (.01$10 \mathrm{mM}$ ), without changing the voltage dependence of activation of IC sodium channels, and this block effect of salicylate could be reversible. The blockade of salicylate might result from salicylate interaction with the resting state of the channel. Although salicylate might bind to the open state of the channel, it did not affect the voltage dependence of 
activation state. The half-maximum activation voltage and the slope factor of the conductance-voltage curve did not differ before and after treatment with $1 \mathrm{mM}$ salicylate, the conductance-voltage relationship was not shifted along the voltage axis, and the time to peak of $I_{\mathrm{Na}}$ was not affected.

On the other hand, salicylate had effects on the inactivation of voltage-gated sodium channels. $1 \mathrm{mM}$ salicylate shifted the voltage dependence of inactivation for sodium channels in the hyperpolarizing direction, but as mentioned before, did not shift the activation toward any direction. Therefore, salicylate molecules had a higher affinity for the inactivation state than for the resting state of the channels. In addition, salicylate delayed the recovery from inactivation of voltage-gated sodium channels of the IC neurons compared to control conditions by increasing the slow time constant. This study also showed that the blocking effects of salicylate on voltage-gated sodium channels could be reversed with a salicylate-free solution. It is well known that many local anesthetics such as lidocaine can induce tinnitus (Brown et al., 1981). They are voltagegated sodium channel blockers. Thus, the mechanism of tinnitus induction may also be related to its blockade of voltage-gated sodium channels. In summary, salicylate exhibited a concentration-dependent sodium channel attenuating effect and interacted with the inactive state of the channel. These effects are consistent to its effect on tinnitus.

\subsubsection{Effects of salicylate on voltage-gated potassium channels}

Liu and Li (2004), also studied the effects of salicylate on the transient outward potassium current $\left(I_{\mathrm{K}(\mathrm{A})}\right)$ and the delayed rectifier potassium current $\left(I_{\mathrm{K}(\mathrm{DR})}\right)$ in acutely dissociated rat inferior colliculus neurons by the whole-cell voltage-clamp method. Potassium channels are widely expressed throughout the brain, and they play crucial roles 
in regulating a variety of cellular processes in neurons, such as setting the resting membrane potential, action potential duration, delay between a stimulus and first action potential, and discharge patterns. The transient outward potassium channel current $\left(I_{\mathrm{K}(\mathrm{A})}\right)$ and the delayed rectifier potassium channel current $\left(I_{\mathrm{K}(\mathrm{DR})}\right)$ are two main potassium channel currents, which have been identified in IC neurons by their activation and inactivation voltage ranges and kinetics and pharmacological sensitivities (Sivamakrishnan and Oliver, 2001). Changes in the function of these potassium channels directly affect neuronal activity, for example, the resting potential, repolarization, and the shape and firing rate of the action potential. $I_{\mathrm{K}(\mathrm{A})}$ determines spike repolarization and influences neuronal excitability and action potential firing. Substances that affect $I_{\mathrm{K}(\mathrm{A}) \text {, }}$ such as 4-AP (4-aminopyridine), prolong the action potentials, which increase the transmitter release and neuronal excitability. $I_{\mathrm{K}(\mathrm{DR})}$ is responsible for the neuronal firing pattern and influences neuronal excitability and synaptic transmission. The inhibition of $I_{\mathrm{K}(\mathrm{DR})}$ by TEA (tetraethylammonium), for example, might depolarize the neuron and result in enhancing the release of neurotransmitters.

In Liu and Li study (2004), salicylate blocked $I_{\mathrm{K}(\mathrm{A})}$ and $I_{\mathrm{K}(\mathrm{DR})}$ in a concentration dependent manner, and decreased the current amplitude of $I_{\mathrm{K}(\mathrm{A})}$ and $I_{\mathrm{K}(\mathrm{DR})}$. However, its blocking potency was much stronger on $I_{\mathrm{K}(\mathrm{DR})}$ than on $I_{\mathrm{K}(\mathrm{A})}$. These results suggested that salicylate inhibited $I_{\mathrm{K}(\mathrm{A})}$ without affecting its activation and inactivation kinetics. On the other hand, besides inhibiting the current amplitude of $I_{\mathrm{K}(\mathrm{DR})}$, salicylate significantly affected its kinetic properties, including shifting the steady-state activation and inactivation curves to the negative direction. 
There are several potential explanations for these results. One possible mechanism is that the number of delayed rectifier potassium channels in IC neurons decreases after salicylate application. Another possibility is that channel properties are altered by salicylate. The hyperpolarizing shifts of half-activation and half-inactivation potential observed for $\mathrm{I}_{\mathrm{K}(\mathrm{DR})}$ after salicylate application indicate that the decrease in $I_{\mathrm{K}(\mathrm{DR})}$ might be responsible for the increased excitability seen in IC neurons following salicylate application, and alterations in potassium channel biophysics, rather than channel number, are responsible for this increment.

The results from Liu and Li (2004) study suggest that salicylate might increase neurotransmitter release, modify the neuronal synaptic transmission and neuronal excitability through blocking the potassium channels. In addition, the potassium current is crucial to repolarization of the action potential. Since $I_{\mathrm{K}(\mathrm{A})}$ is transient, the

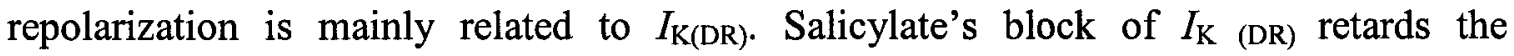
repolarization. These results are consistent with the previous reports that salicylate induces an upregulation of the neuronal spontaneous activity (Jastreboff and Sasaki, 1986 ) and of the metabolic activity using $2 \mathrm{DG}$ as a marker in the IC. However, Liang and Zhong (2002) found that after sodium salicylate $(1 \mathrm{mM})$ administration, outward potassium channel current in outer hair cells isolated from guinea pig cochlea underwent transient increase and then declined. Thus, the effects of salicylate on voltage-gated potassium channels in IC neurons are not identical with its effects on voltage-gated potassium channels in outer hair cells. Considering Liu and Li (2004) study, it could be concluded that voltage gated potassium channels, especially delayed rectifier potassium channels, may play an important role in the mechanisms of salicylate-induced tinnitus. 


\subsubsection{Effects of salicylate on voltage-gated calcium channels}

Effects of sodium salicylate on L-type voltage gated calcium channels also have been studied by Liu et al. (2005) in the freshly dissociated inferior colliculus neurons of rats by the whole-cell voltage clamp method. Both neonatal and adult rat IC neurons express low, mid, and high threshold voltage-activated calcium channels. High voltageactivated calcium channels comprise L-, N-, P/Q and R-type calcium channels based on their sensitivity to various calcium channel ligands. L-type calcium channels mediate long-lasting calcium currents in response to depolarization in excitable cells. Calcium influx through L-type calcium channels leads to activation of a cascade of intracellular signals and is responsible for the after hyperpolarization phase following action potentials in neurons (Marrion and Tavalin, 1998). In addition, calcium currents through L-type calcium channels contribute more to regulating neurotransmitter release than do other types of calcium currents, including GABA ( $\gamma$-aminobutyric acid) release (Murakami et al., 2002). This particular feature of L-type calcium channel-dependent calcium influx is thought to play a key role in neuronal excitability and plasticity. Liu and Li (2005) study indicated that the amplitudes of calcium current through L-type voltage-gated calcium channels decreased with increments of salicylate concentrations and this block effect of salicylate on $I_{\mathrm{Ca}, \mathrm{L}}$ could be reversible. Salicylate $(1 \mathrm{mM})$ decreased $I_{\mathrm{Ca}, \mathrm{L}}$, and changed the voltage dependence of calcium current activation, but did not significantly alter the steady-state activation kinetics of $I_{\mathrm{Ca}, \mathrm{L}}$. However, salicylate significantly shifted the steady-state inactivation curve in the hyperpolarizing direction, and significantly delayed the $I_{\mathrm{Ca}, \mathrm{L}}$ recovery course from inactivation kinetics. 
Voltage-gated calcium channels are known to contribute to the release of various neurotransmitters, including GABA. GABA is the most common inhibitory neurotransmitter and is involved in inhibiting IC neurons. The decrease in GABA level might be responsible for the increased activity of the IC neurons. L-type calcium channels contribute to GABAergic synaptic facilitation by the activating second messenger system (Jensen and Mody, 2001) and/or by increasing intracellular calcium concentration (Jensen et al., 1999). Thus, salicylate-induced attenuation of $I_{\mathrm{Ca}, \mathrm{L}}$ may decrease the GABA release within the IC by inhibiting the second messenger system and/or reducing calcium concentration in the neurons.

Salicylate alters auditory processing within the IC by depressing the L-type calcium channels, which results in loss of GABA mediated inhibition and might be the cause of the development of tinnitus. This hypothesis might explain why the exogenous calcium supplement, which could enhance the intracellular calcium concentration in the IC neurons and then increase GABA release, attenuates the manifestation of tinnitus, and eliminates the salicylate-induced increase of average activity and burst activity of single IC neurons (Chen and Jastreboff, 1995).

In all, voltage-gated channels may play a critical role in mechanisms of salicylateinduced tinnitus. However, the relations among the effects of salicylate on calcium channels, sodium channels and potassium channels are still obscured and need further study. 


\section{Purpose of the study}

Tinnitus is as an auditory perception without an external auditory source and it is associated with abnormalities in spontaneous neural activity in the auditory system, changes in the timing of neural discharges (i.e., the temporal firing properties of neurons), and an increase in bursting activity of neurons. Despite the abundance of assumed locations of the generator of tinnitus, most current hypotheses agree that abnormal neural activity is interpreted and perceived as tinnitus in higher auditory centers. The IC is a major structure in the central auditory system and plays a crucial role in processing inputs from both the ascending and descending pathways. It has been suggested that $\mathrm{IC}$ is one of the candidates for involvement in generation of tinnitus.

Ototoxic drugs like salicylate and quinine are one of the important causes of tinnitus. Salicylate is a well-known substance to produce reversible tinnitus in humans and animals. However, the neural origins and mechanisms of salicylate-induced tinnitus generation in the central auditory pathway have not yet been fully clarified. The purpose of the current study was to investigate the effects of salicylate on intrinsic membrane properties of neurons in the central nucleus of the inferior colliculus (ICC). This study might provide a better understanding of the mechanisms of salicylate-induced tinnitus. Furthermore, this study could give an insight into how salicylate affects signal processing in ICC neurons.

The method of the proposal study was whole-cell patch clamp recordings from the inferior colliculus neurons in the rat brain slice. Once a whole-cell patch was made successfully, membrane properties of the neuron were examined under current clamp mode. The previous in vitro studies about the effects of sodium salicylate on IC neurons 
employed voltage-clamp recoding to examine the effects of salicylate on specific voltagegated sodium, potassium and calcium channels (Liu and Li, 2004; Liu et al., 2005). However, the present study investigated the effects of sodium salicylate on intrinsic membrane properties of individual ICC neurons instead of specific types of ion channels, on ICC neurons. Furthermore, the procedure for neuron isolation used in the studies on isolated ion channels did not allow identification of the neuron's location within the IC, i.e., ICC, ICD and ICX. In this study, only ICC neurons were examined in IC brain slice preparation. Electrical membrane properties investigated in the current study included resting membrane potential, firing patterns and firing abilities, i.e., threshold and frequency of firing, action potential (AP) properties such as, AP amplitude and duration, afterhyperpolarization (AHP) amplitude and duration, and responses to hyperpolarization. The current-voltage relationship, membrane input resistance and time constant were also determined.

\section{Materials and methods}

\subsection{Brain slice preparation}

Young Wistar rats of either sex aged 9-15 days (Charles River Co., Quebec, Canada) were used for this study. All procedures involving the use of animals in this study were carried out in accordance with the guidelines of Canadian Council on Animal Care and were approved by the animal Care Committee of Carleton University. All efforts were made to use only the number of animals necessary to produce reliable results. The rats were anaesthetized by isofluorane inhalation and were decapitated and their brains were removed. After the removal, the brain was placed in artificial 
cerebrospinal fluid (ACSF) at room temperature $\left(20^{\circ} \mathrm{C}-22^{\circ} \mathrm{C}\right)$ with the following composition (in mM): $129 \mathrm{NaCl}, 3 \mathrm{KCl}, 1.2 \mathrm{KH}_{2} \mathrm{PO}_{4}, 2.4 \mathrm{CaCl}_{2}, 1.3 \mathrm{MgSO}_{4}, 20$ $\mathrm{NaHCO}_{3}, 3 \mathrm{~N}-2$-hydroxyethylpiperazine-N-2-ethanesulfonic acid (HEPES) and 10 glucose in dionized water, which was saturated with $95 \% \mathrm{O}_{2}-5 \% \mathrm{CO}_{2}$. The pH of ACSF was measured during making the solution before each experiment, and was adjusted with concentrated $\mathrm{HCl}$ or $\mathrm{NaOH}$ to $7.35-7.45$, if it was required.

The auditory midbrain was blocked and mounted on the stage of a tissue slicer using cyanoacrylate adhesive and dissected in oxygenated ACSF and brain slices (300$350 \mathrm{~mm}$ thick) was cut in the frontal plane through the midbrain with a tissue brain slicer. A slice containing the ICC, ICD and ICX was selected and transferred to a recording chamber. The volume of the chamber was about $1.5 \mathrm{ml}$ and the slice was held securely in the recording chamber with two pieces of nylon mesh. An oxygenated ACSF solution was circulated continuously through the recording chamber and around the brain slice during the course of the experiment at a flow rate of 6-8 $\mathrm{ml} / \mathrm{min}$. The ACSF solution was saturated with $95 \% \mathrm{O}_{2}-5 \% \mathrm{CO}_{2}$ before it was circulated through the slice chamber and its temperature was kept at $28-30^{\circ} \mathrm{C}$. The slice was kept in the recording chamber for approximately twenty minutes before any recording was made.

\subsection{Whole-cell patch clamp recording}

The brain slice chamber was illuminated from below by light passed through a Zeiss microscope. The outline of the ICC was identified. Then a blind patch procedure was used for whole-cell recording in current clamp mode with an Axopatch 200A amplifier (Axon Instruments) and the data was analyzed with pclamp8 software (Axon 
Instruments). The patch pipettes with tip diameter of 1-2 $\mu \mathrm{m}$ were pulled from thinwalled glass tubing (Kimbax-51, Kimble), using a Narishige PP-830 vertical puller (Narishigie, Japan). The patch electrodes resistance was between 4-7 M $\Omega$, and they were filled with internal solution of the following composition (in $\mathrm{mM}$ ): $130 \mathrm{~K}$ gluconate, 2 $\mathrm{MgCl}_{2}, 5 \mathrm{KCL}, 0.3 \mathrm{GTP}, 2 \mathrm{ATP}, 0.6$ EGTA, 10 HEPES. The internal solution osmolarity was $300 \mathrm{mOsm}$ and $\mathrm{pH}$ was 7.25 . The internal solutions were filtered with $4 \mathrm{~mm}$ nylon syringe filters $(0.22 \mu \mathrm{m}$ pore size $)$.

A single recording electrode was placed into the ICC. The blind whole-cell patch was achieved utilizing the blow and suck procedure. First, the recording electrode was positioned over the ICC and then was lowered with a gross three-dimensional manipulator towards the surface of the ICC. While being lowered, positive pressure was applied by mouth through the electrode holder to prevent the electrode from being clogged by tissue or debris. After the electrode touched the tissue there was an increase in the impedance of the electrode. The electrode was then finely advanced into the tissue using a Narishige hydraulic three-dimensional micromanipulator. Once the tip of the electrode hit the neuron, a gigaohm (G $\Omega$ ) seal was formed. Waiting for about one minute after achieving a gigaohm seal was helpful in allowing some further stabilization of the seal. A whole-cell patch configuration was achieved by applying a sudden negative pressure by mouth through the electrode holder, tearing the cell membrane.

As mentioned before, an Axopatch 200A amplifier was used for the whole-cell patch clamp recordings. Upon achieving the whole cell configuration, capacity transient was canceled and series resistance (typically 15-25 M 2 ) was monitored throughout the experiment and it was compensated by $70 \%$. If a change of the series resistance was 
more than $15 \%$ of the initial value, the recording was terminated. In addition, the resting membrane potential had to be relatively stable staying at the same level with little fluctuation during the period of recording. The liquid junction potential was calculated and used to correct for the recorded membrane potential. Signals were filtered at $5 \mathrm{KHz}$ and were digitized at $5-10 \mathrm{kHz}$ by Digidata $1320 \mathrm{~A}$ interface, were acquired by pClamp 8 and were analyzed by pClamp8 (Axon Instruments) on an IBM-compatible PC.

After a whole-cell patch configuration was made successfully, membrane properties were examined under current clamp mode. The drug that was applied in this study was sodium salicylate. For evaluation of salicylate effects, sodium salicylate was dissolved in extracellular solution and was applied to the cells using a perfusion application system. A fresh solution of the sodium salicylate was made every day. Different concentrations of salicylate were applied for experiments $(1,2,3,4,5$ and 10 $\mathrm{mM})$. The drug was dissolved in ACSF to the desired final concentration and was kept in separate oxygenated container that was connected to the main perfusion channel. The $\mathrm{pH}$ of the drug solution was checked before the application and was adjusted to 7.4 , if it was necessary. The drug was applied to the brain slice by switching the perfusing solution from ACSF to the drug solution. All chemicals were purchased from Sigma Chemical Co. (St. Louis, MO)

After making the whole-cell configuration, the amplifier was set to operate in the current clamp mode to investigate the intrinsic membrane properties of ICC neurons. The response of each neuron was examined to pulse current injections with four protocols, i.e., "standard", “prehyperpolarization", “depolarization", and "long”. In "standard" and "long" protocols, currents were injected for 200 or 300 ms respectively, over the range of 
-300 to $300 \mathrm{pA}$ in $20 \mathrm{pA}$ increment/step. In "depolarization" protocol, the cell also was subjected to a range of positive current injection from 0 to $400 \mathrm{pA}$ for $200 \mathrm{~ms}$, which was used to test the firing pattern and the firing ability of the neuron. In order to classify further the firing pattern, neurons were examined by a prehyperpolarization protocol, which included the injection of a "prehyperpolarization" current of -200 pA for $200 \mathrm{~ms}$, prior to depolarizing current injections that had a range of 0 to $400 \mathrm{pA}$ in $20 \mathrm{pA}$ increment/step for $300 \mathrm{~ms}$.

\subsection{Data analysis}

The resting membrane potential, input resistance, time constant, firing rate, threshold of firing, properties of action potentials (APs) including amplitude, half-width, AHP amplitude and duration were examined. The change in membrane potential elicited by intracellular injection of negative current was obtained by measuring the voltage between the baseline membrane potential and the peak hyperpolarization. These values were then used to plot the current-voltage (IV) curves. From the curve, the slope was calculated from a range of values that were approximately linear. This slope represented input resistance $\left(R_{\text {in }}\right)$ of the cell membrane, measured in megaohm $(M \Omega)$. The time constant $(\tau)$ of the cell membrane was obtained by measuring the time taken membrane potential to reach $63 \%$ of the final voltage in response to a hyperpolarizing current pulse of - $300 \mathrm{pA}$.

The threshold current for firing was defined as the lowest level of positive current injection required for eliciting one action potential. All analyses of APs including amplitude, half-width, and afterhyperpolarization (AHP) were performed on action 
potentials elicited by the threshold current. The amplitude of the AP was measured from the base of the spike to its peak. The measurements for action potential half-width were taken at 50\% amplitude of the spike. The AHP amplitude was measured from the beginning of the AP to the maximum deviation of the AHP (Fig. 12). The AHP halfwidth was measured at $50 \%$ of the maximum hyperpolarization following the AP.

When neurons displayed AP trains in response to positive current larger than the threshold current, the firing frequency was calculated, and rate level function (RLF) was plotted. The RLF was examined with a range of current injections from 0 to $400 \mathrm{pA}$ for a duration of $200 \mathrm{~ms}$. The firing frequency was calculated as hertz $(\mathrm{Hz})$, i.e., $\mathrm{X}$ spike/sec, by multiplying the number of APs that were elicited during the current pulse by 5 .

The neuron's firing pattern was determined by responses to depolarizing current injections. The cell was subjected to a range of positive current injection from 0 to 400 pA for $200 \mathrm{~ms}$. When a train of APs was elicited, the neuron was categorized as regulartype, adaptation-type or pauser-type based on the appearance of its firing pattern. In regular cells, the interspike interval was uniform or equal throughout the spike train. In adaptation neurons, the first interspike interval had a shorter duration than the subsequent interspike intervals. Furthermore, the last interspike interval must be at least $50 \%$ longer than the first interspike interval in that spike train. For pauser neurons, the first interspike interval had a longer duration than the subsequent interspike intervals. In these pauser cells, an initial pause should be followed by a sustained train of APs with shorter, regular interspike intervals. The firing pattern was verified at two levels of depolarizing current injection that elicited 4 to 6 APs. The classification of pauser-type cell was also verified by subjecting the neuron to a fixed prehyperpolarizing current injection ( $-200 \mathrm{pA})$ 
followed by varying levels of depolarizing current injection ( 0 to $400 \mathrm{pA}$ ). If the pause was still evident in these traces, the cell was categorized as pauser-type.

The data were analyzed using Clampfit module in pClamp 8.0 software. Statistical analyses were conducted with SPSS 11.5, using repeated measure ANOVA with post hoc. Figures were made using Microsoft Excel 2002. Averaged data were presented as mean $\pm \mathrm{SE}$.

\section{Results}

The data presented here are based upon whole cell patch-clamp recordings from 87 ICC neurons. Measurements of resting membrane potential (RMP), input resistance $\left(R_{\text {in }}\right)$, time constant $(\tau)$, rate level function, AP and AHP properties were made from randomly chosen samples in these cells (see Table 1).

\subsection{Effects of salicylate on passive membrane properties}

\subsubsection{Resting membrane potential}

The membrane potential of a cell at rest was measured before and during application of salicylate $(1 \mathrm{mM}$ to $5 \mathrm{mM})$. The range of RMP of ICC neurons encountered was between $-56.5 \mathrm{mV}$ to $-65.3 \mathrm{mV}$ with the brain slice in ACSF. The RMP measurement revealed no significant change following salicylate use in comparison to control condition. The mean RMP was $-62.9 \pm 0.5 \mathrm{mV}$ before, and $-63.8 \pm 0.6 \mathrm{mV}$ during salicylate application $(\mathrm{n}=25)(\mathrm{p}>0.05)$. 


\subsubsection{Current-voltage relationship and input resistant}

By passing hyperpolarizing current pulses into a cell, the relationship between the current (I) and the resulting changes in membrane potential (V) was determined. The I-V curve was obtained by plotting the steady state voltage against the injected current. Figure 1A and 1B illustrate traces recorded from an ICC neuron in response to 3 levels of negative current injection (-300, -200, $-100 \mathrm{pA}$ ) before (Fig. 1A) and during salicylate application (Fig. 1B). The current-voltage relationships of this neuron (Fig. 1C) and 27 ICC neurons (Fig. 1D) tested were approximately linear and were not changed by 1 to 5 $\mathrm{mM}$ salicylate. Using the linear portion of the I-V curve, the slope was calculated as $R_{\mathrm{in}}$, which did not show significant change following salicylate application. The mean $R_{\text {in }}$ was 269.1 $\pm 14.9 \mathrm{M} \Omega$ before, and 282.0 $\pm 16.2 \mathrm{M} \Omega$ during salicylate application $(n=27)(\mathrm{p}>$ $0.05)$.

\subsubsection{Time constant}

The time constant $(\tau)$ of the tested neurons was calculated from the responses to negative current injection. There was no significant difference in time constant before and during 1 to $5 \mathrm{mM}$ salicylate application. The mean $\tau$ was $7.80 \pm 0.50 \mathrm{~ms}$ before, and $7.47 \pm 0.51 \mathrm{~ms}$ during salicylate application $(\mathrm{n}=29)(\mathrm{p}>0.05)$

\subsection{Effects of salicylate on membrane excitability}

\subsubsection{Firing pattern}

Three firing patterns were detected in the examined neurons: regular-type, adaptation-type and pauser-type. Figure $2 \mathrm{~A}$ shows traces recorded from three representative neurons in response to depolarizing current injections. The left upper and 
lower traces in Figure 2A show responses to depolarizing current injection (220 pA) from a regular-type neuron before and during salicylate application. The interspike intervals were uniform or regular throughout the spike train in this neuron. During salicylate application $(5 \mathrm{mM})$, the uniform intervals were maintained.

The middle upper and lower traces in Figure 2A show spike trains in response to depolarizing current injection $(180 \mathrm{pA})$ from an adaptation-type neuron before and during salicylate application $(5 \mathrm{mM})$. The first interspike interval had a shorter duration than the subsequent interspike intervals. The neuron seemed to adapt to the current injection, and the last interspike interval was 1.5 times longer than the first one. After using salicylate, although all the intervals were longer than those in control condition, the neuron retained the adapting firing pattern. Increment of interspike intervals and changes in AP shape during drug application may be due to the effect of salicylate on firing frequency, which will be described in next section.

The right upper and lower traces in Figure $2 \mathrm{~A}$ demonstrate responses to depolarizing current injection (160 pA), following hyperpolarizing current injection (-200 pA) from a pauser-type neuron before and during salicylate application. The traces indicate that the first interspike interval was longer (pause) than the subsequent interspike intervals, and this pause was followed by a sustained spike train with shorter, but regular interspike intervals. Following salicylate application ( $4 \mathrm{mM})$, all the intervals became longer. However, the firing pattern remained the same as in control condition.

Figure 2B illustrates the distribution of tested ICC neurons based on their firing patterns $(n=26)$ 


\subsubsection{Firing abilities}

The ICC neurons encountered were subjected to varying levels of positive intracellular current injection up to $400 \mathrm{pA}$, in order to assess a cell's ability of firing APs. The responses also revealed the threshold of current injection required to initiate an $\mathrm{AP}$ and the threshold of membrane potential at which an AP was elicited in the neuron.

\subsubsection{Threshold of firing}

The membrane potential threshold for firing was defined as a level of a neuron's membrane potential at which the neuron responded to depolarizing current injection with only one AP (Fig. 3A, arrows). The current threshold for firing was defined as the lowest level of positive current injection which elicited only one action potential. Figure 3A demonstrates responses of a neuron with the threshold current injection before and during salicylate application, and after wash. The current threshold for firing decreased during salicylate application (from $140 \mathrm{pA}$ to $120 \mathrm{pA}$ ), and returned to the same level after washing out the drug. Figure $3 \mathrm{~A}$ also indicates the membrane potential threshold before and during drug application, and after wash (control: - $31.5 \mathrm{mV}$, drug: - $35 \mathrm{mV}$, wash: $33 \mathrm{mV}$ ). ICC neurons $(\mathrm{n}=15)$ displayed a significantly lower current threshold during salicylate application as compared to control condition (Fig. 3B). The mean threshold current was $100.0 \pm 11.6 \mathrm{pA}$ before, and $82.7 \pm 11.0 \mathrm{pA}$ during salicylate application $(\mathrm{p}=$ 0.004).

Figure $3 \mathrm{C}$ indicates the average of the membrane potential threshold of the same group of ICC neurons as shown in Figure 3B before and during salicylate application, and after wash. The mean membrane potential threshold $(-40.0 \pm 2.4 \mathrm{mV})$ was also significantly lower than that in control condition $(-36.8 \pm 2.3 \mathrm{mV})(\mathrm{p}=0.001)$. 


\subsubsection{Firing frequency}

Firing frequency or rate level function was reflected by number of action potentials during depolarizing current injection. Firing frequency of all the examined neurons with sustained regular, sustained adapting or pauser pattern was affected by salicylate. Figure 4 illustrates traces of spike trains of a neuron that was injected with depolarizing current injections (from bottom to top: 100, 260 and $400 \mathrm{pA}$ ) before and during salicylate application ( $2 \mathrm{mM})$, and after wash. There was no obvious change in spike number and firing frequency during salicylate application at a lower level (100 pA) of depolarizing current injection (lower traces in Fig. 4A and 4B). By increasing the level of depolarizing current injection, the number of spikes and firing frequency increased in control condition (middle and upper traces in Fig. 4A). However, during salicylate application, the number of spikes decreased, and the APs became wider and smaller (middle and upper traces in Fig. 4B) compared to those elicited at the same levels of current injection in control condition. These effects were more evident at higher levels of current injection. Only a few APs and several small humps were generated in response to $400 \mathrm{pA}$ current injection (upper trace in Fig. 4B). The firing ability of the neuron was completely recovered after wash (Fig. 4C), indicating that the effects of salicylate on AP firing were reversible. Figure $5 \mathrm{~A}$ shows changes of firing frequency as a function of current level before and during salicylate, and after wash for the neuron shown in Figure 4. The firing rate was slightly more at lower levels (40 to $260 \mathrm{pA}$ ) of depolarizing current injection during salicylate application as compared to control condition. However, at higher levels ( 280 to $400 \mathrm{pA}$ ) of current injection there was a sharp decrease in firing frequency during drug exposure. Figure 5B shows the average firing frequency for all the 
tested ICC neurons $(\mathrm{n}=11)$, before and during 1-2 $\mathrm{mM}$ salicylate. There was a decrease in the firing frequency of these cells during salicylate application as compared with in control condition at levels of $300-400 \mathrm{pA}$. The average firing frequency of the ICC neurons was $49 \pm 6.9 \mathrm{~Hz}(300 \mathrm{pA})$ and $59.1 \pm 7.5 \mathrm{~Hz}(400 \mathrm{pA})$ in control condition, and $32.3 \pm 6.2 \mathrm{~Hz}(300 \mathrm{pA})$ and $36.8 \pm 6.6 \mathrm{~Hz}(400 \mathrm{pA})$ in drug condition (Fig. 5B).

Figure 6 shows responses of another ICC neuron that was injected with depolarizing current injections (from bottom to top: 200, 300 and $400 \mathrm{pA}$ ) before and during salicylate application $(5 \mathrm{mM})$, and after wash. There was an increment in spike number by increasing the level of current injection in control condition, e.g., 5 spikes at $200 \mathrm{pA}$ to 8 spikes at 300 pA (lower and middle traces in Fig. 6A). Further increment in spike number was observed (10 spikes) at a level of 400 pA (upper trace in Fig. 6A). Following salicylate application the number of APs, even at lower level of current injection (200 pA) greatly decreased (Fig. 6B). Decreasing in spike amplitude and widening of APs were also observed. These changes were more obvious at higher levels of current injection (middle and upper traces in Fig. 6B), but reversible after wash (Fig. 6C). Figure 7A shows the RLF of the same neuron as shown in Figure 6. There was a marked reduction in firing frequency following application of salicylate as compared to control condition in response to depolarizing current injection from 200 to $400 \mathrm{pA}$. Figure $7 \mathrm{~B}$ illustrates the average firing frequency for all the neurons $(n=17)$ that were subjected to 3-5 $\mathrm{mM}$ salicylate application. There was a decrease in the firing frequency of these cells during salicylate application as compared with control condition at current injection of 200 to $400 \mathrm{pA}$. The average firing frequency of the ICC neurons tested was $31.5 \pm 4.1 \mathrm{~Hz}(200 \mathrm{pA})$ and $56.2 \pm 5.7 \mathrm{~Hz}(400 \mathrm{pA})$ in control condition, and $19.4 \pm 3.8$ 
$\mathrm{Hz}(200 \mathrm{pA})$ and $27.9 \pm 5.3 \mathrm{~Hz}(400 \mathrm{pA})$ in drug condition $(3-5 \mathrm{mM})$. It seemed that when the salicylate dose increased (from 1-2 to $3-5 \mathrm{mM}$ ), the reduction of the firing frequency was more prominent.

The effect of salicylate on the firing frequency of the pauser-type neurons was similar to those showed in sustained-regular and sustained-adaptation neurons. Figure 8 A shows a neuron with a typical pauser pattern in response to injection of $300 \mathrm{pA}$ depolarizing current following a current pulse of $-200 \mathrm{pA}$. The first interspike interval (pause) was longer than subsequent ones. During application of salicylate, the first interspike interval greatly decreased and became shorter than subsequent ones in response to the same level of depolarizing current pulse as in control condition (Fig. 8B). Thus, the pause pattern was no longer detected. The neuron fired only one regular AP at the onset of the current pulse. The subsequent APs showed progressive decrease in their amplitude, and a few small humps were generated afterwards. These changes were reversible (Fig. 8C) after washing out the drug.

Figure 9 shows changes in the first interspike interval with increase in depolarizing injection from 200 to 400 pA for the neuron shown in Figure 8 under control and drug conditions. The interval decreased from $47.3 \mathrm{~ms}$ to $27.1 \mathrm{~ms}$ in control condition, and from $38.5 \mathrm{~ms}$ to $7.4 \mathrm{~ms}$ during salicylate application. These results indicated that salicylate decreased the pause more at higher (300-400 pA) than at lower levels (200-300 pA) of depolarizing current injection. Therefore, the pauser-type of firing pattern could not be detected at higher levels (300 to $400 \mathrm{pA}$ ).

The effect of salicylate on firing frequency of 28 ICC neurons is summarized in Figure 10. Figure 10A shows the average frequency for all concentrations (1-5 mM) of 
salicylate used in these experiments. Salicylate significantly decreased the firing frequency at levels of current injection from 240 to $400 \mathrm{pA}(\mathrm{p}=0.01)$. This effect was reversible. Figure $10 \mathrm{~B}$ indicates that at lower levels of current injection from 20 to 180 $\mathrm{pA}$, there was an increase in firing frequency by increasing the level of current injection in control, drug and wash conditions. The average firing frequency range was from $0.4 \pm$ $0.2 \mathrm{~Hz}(20 \mathrm{pA})$ to $26.4 \pm 3.1 \mathrm{~Hz}(180 \mathrm{pA})$ in control condition, and $0.4 \pm 0.2 \mathrm{~Hz}(20 \mathrm{pA})$ to $21.6 \pm 3.1 \mathrm{~Hz}(180 \mathrm{pA})$ in drug condition. The average firing frequency for levels between 20 to $180 \mathrm{pA}$ was $11.0 \pm 1.9$ in control condition, and $11.0 \pm 2.0$ during drug application. These results indicate that at 20-180 pA levels of current injection, the increase in firing frequency was similar in control and drug conditions. Figure 10C shows that the increase in firing frequency differed in control, drug and wash conditions. The average firing frequency range was from $30.9 \pm 3.2 \mathrm{~Hz}(200 \mathrm{pA})$ to $57.3 \pm 4.5 \mathrm{~Hz}(400$ $\mathrm{pA})$ in control condition, and from $23.9 \pm 3.2 \mathrm{~Hz}(200 \mathrm{pA})$ to $31.4 \pm 4.1 \mathrm{~Hz}(400 \mathrm{pA})$ in drug condition. The average of firing frequency for levels between 200 to $400 \mathrm{pA}$ was $46.0 \pm 4.0 \mathrm{~Hz}$ in control condition, and $28.4 \pm 3.7 \mathrm{~Hz}$ during salicylate application. These results indicate that at the higher levels of current injection (200 to $400 \mathrm{pA}$ ), there was only a slight increase in firing frequency with increasing levels of current injection during drug condition in comparison to control and wash conditions. In summary, salicylate decreased firing frequency of ICC neurons at high levels $(200-400 \mathrm{pA})$ of depolarizing current injection, but did not change the firing frequency significantly at low levels $(20-$ $180 \mathrm{pA}$ ) as compared to control condition.

The degree of the reduction in firing frequency of the ICC neurons was dependent on how sensitive the neurons were to salicylate. Figure 11 illustrates the proportion of the 
ICC neurons that had a reduction in firing rate from $5.9 \%$ to $94 \%$ at the level of current injection of $400 \mathrm{pA}$ during salicylate application of 2 to $5 \mathrm{mM}$ as compared to control condition. The results suggest a large variation in sensitivity of these neurons to salicylate $(n=25)$.

\subsection{Effects of salicylate on action potential properties}

\subsubsection{Action potential}

The analysis of the action potential amplitude was performed on 23 neurons that were subjected to salicylate. There was no significant difference in AP amplitude before and during drug application. The mean AP amplitude was $41.6 \pm 2.1 \mathrm{mV}$ before and 40.8 $\pm 2.21 \mathrm{mV}$ during drug exposure $(\mathrm{p}>0.05)$. The action potential half-width following salicylate application also did not display a significant difference before and during drug exposure. The mean AP half-width was $1.26 \pm 0.10 \mathrm{~ms}$ before and $1.32 \pm 0.09 \mathrm{~ms}$ during drug application $(n=15)(p>0.05)$. These results were based on measurements of the action potential at the threshold current level. However, by injecting more depolarizing currents, APs showed changes in amplitude and half-width following salicylate application. These changes included reduction in AP amplitude and widening of APs. The effects were more noticeable at higher levels of depolarizing current injections (Figs 4, 6 and 8). For the neurons with a high sensitivity to salicylate, these changes were observed even at lower levels of depolarizing current injections.

\subsubsection{Afterhyperpolarization}

In most ICC neurons, the action potential was followed by a transient hyperpolarization, so called afterhyperpolarization (AHP) or undershoot. Comparisons of 
AHP amplitude and AHP duration were made before and during salicylate. Figure 12 illustrates an example, showing a decrease in AHP amplitude from $10.4 \mathrm{mV}$ before, to $6.4 \mathrm{mV}$ after salicylate application. The data from 15 neurons indicated a significant reduction in AHP amplitude during salicylate application as compared to control condition. The mean AHP amplitude was $13.6 \pm 1.3 \mathrm{mV}$ before and $11.0 \pm 1.4 \mathrm{mV}$ during drug application $(p=0.002)$. However, there was no significant difference in the AHP half-width before and during salicylate application. The mean AHP half-width duration was $65.7 \pm 6.4 \mathrm{~ms}$ before, and $61.5 \pm 6.6 \mathrm{~ms}$ during drug exposure $(n=15)(p>$ $0.05)$.

\subsubsection{Hyperpolarization-activated current $\left(I_{h}\right)$}

Injections of hyperpolarizing current into ICC neurons led some cells to exhibit an initial hyperpolarization followed by a slowly depolarizing sag toward the resting membrane potential, which reflected the presence of hyperpolarization induced current, $I_{h}$. Figure 13A shows traces recorded from a neuron in response to varying levels of hyperpolarizing current injection (from bottom to top: $-300,-200,-100 \mathrm{pA}$ ). The top trace did not clearly display a depolarizing sag. However, when a sufficient level of hyperpolarization was achieved the sag became evident (bottom trace). The currentvoltage plot (Fig. 13C) showed an essentially linear relationship for the initial hyperpolarization $(\boldsymbol{\Delta})$, but a non-linear current-voltage relationship for the steady-state of voltage changes $(\bullet)$.

Figure 13B shows traces recorded for the same neuron in response to hyperpolarizing current injection following salicylate application. There was a little difference in the magnitude of the depolarizing sag during drug application compared to 
control condition (18.6 mV in control condition, $18.0 \mathrm{mV}$ during drug exposure). The I-V curves for the initial hyperpolarization and steady state of voltage changes in control condition and after exposure to salicylate were essentially the same (Fig. 13C). Figure 13D shows the I-V curves for the mean initial hyperpolarization and steady state hyperpolarization for 4 neurons before and during salicylate application. The data indicated no significant difference in the depolarizing sag for these two conditions.

\subsubsection{Rebound after hyperpolarization}

In some of the ICC neurons encountered, a broad rebound depolarization was elicited following hyperpolarizing current injections. In order to investigate the effect of salicylate on the rebound, the amplitude of the depolarizing rebound after hyperpolarization were measured. Figure 14 shows traces that consisted of a hyperpolarization and a depolarizing rebound following a hyperpolarizing current injection $(-120 \mathrm{pA})$ in control condition and during drug exposure in a neuron. There was no obvious change in the rebound amplitude $(13 \mathrm{mV})$ following salicylate application as compared to control condition. 
Table 1 - The effects of salicylate on intrinsic properties of the ICC neurons

\begin{tabular}{|c|c|c|c|c|c|c|c|c|c|}
\hline & \multicolumn{3}{|c|}{ Control } & \multicolumn{3}{|c|}{ Drug } & \multicolumn{3}{|c|}{ Wash } \\
\hline & Mean & S.E. $( \pm)$ & $\mathrm{n}$ & Mean & S.E. $( \pm)$ & $\mathrm{n}$ & Mean & S.E. $( \pm)$ & $\mathrm{n}$ \\
\hline RMP (mV) & -62.9 & 0.5 & 25 & -63.8 & 0.6 & 25 & -63.5 & 0.5 & 25 \\
\hline $\operatorname{IR}(\mathrm{M} \Omega)$ & 269.1 & 14.9 & 27 & 282.0 & 16.2 & 27 & 287.3 & 16.3 & 27 \\
\hline $\mathrm{TC}(\mathrm{ms})$ & 7.8 & 0.5 & 29 & 7.5 & 0.5 & 29 & 7.6 & 0.6 & 29 \\
\hline $\mathrm{TR}(\mathrm{mV})^{*}$ & -36.8 & 2.3 & 15 & -40.0 & 1.8 & 15 & -36.6 & 2.4 & 15 \\
\hline TR (pA)* & 100.0 & 11.6 & 15 & 82.7 & 11.0 & 15 & 102.7 & 10.7 & 15 \\
\hline AP $\operatorname{amp}(\mathrm{mV})$ & 41.6 & 2.1 & 23 & 40.8 & 2.2 & 23 & 40.5 & 2.5 & 23 \\
\hline AP hw (ms) & 1.3 & 0.1 & 15 & 1.3 & 0.1 & 15 & 1.3 & 0.1 & 15 \\
\hline AHP amp $(\mathrm{mV})^{* *}$ & 13.6 & 1.3 & 15 & 11.0 & 1.4 & 15 & 12.8 & 1.2 & 15 \\
\hline AHP hw (ms) & 65.3 & 6.0 & 16 & 62.1 & 6.2 & 16 & 65.8 & 5.8 & 16 \\
\hline
\end{tabular}

RMP - resting membrane potential; IR - input resistance; TC - time constant; TR - AP threshold; TR - threshold current; AP amp - action potential amplitude; AP hw-action potential half-width; AHP amp - AHP amplitude; AHP hw - AHP half-width; $:$ p $<0.01 ;{ }^{* *}: \mathrm{p}<0.001$ 
올 올

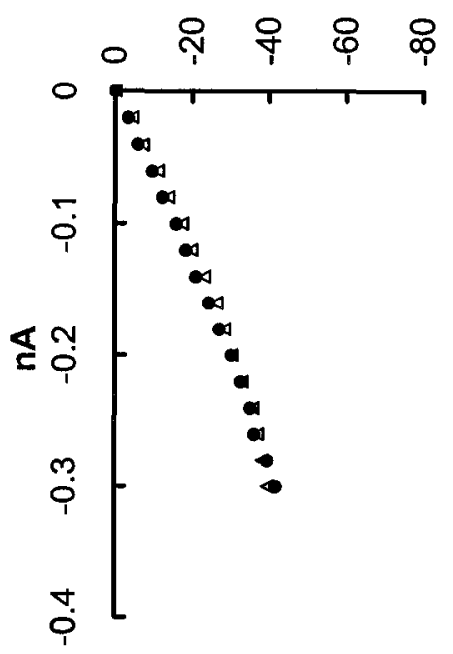

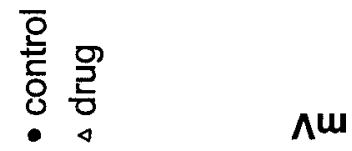

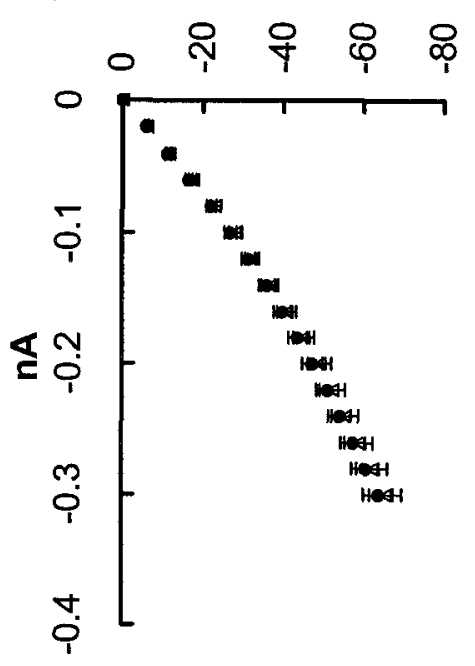

0

흥

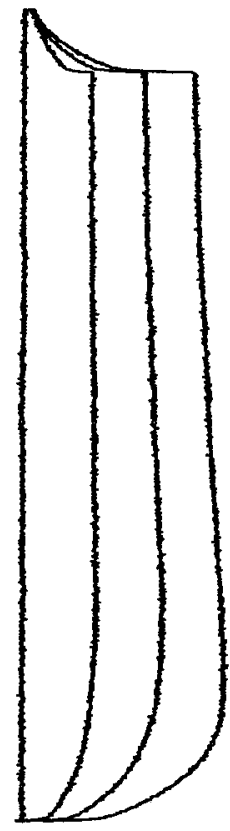

0

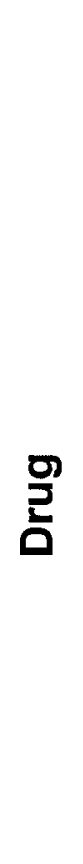

올

กับ

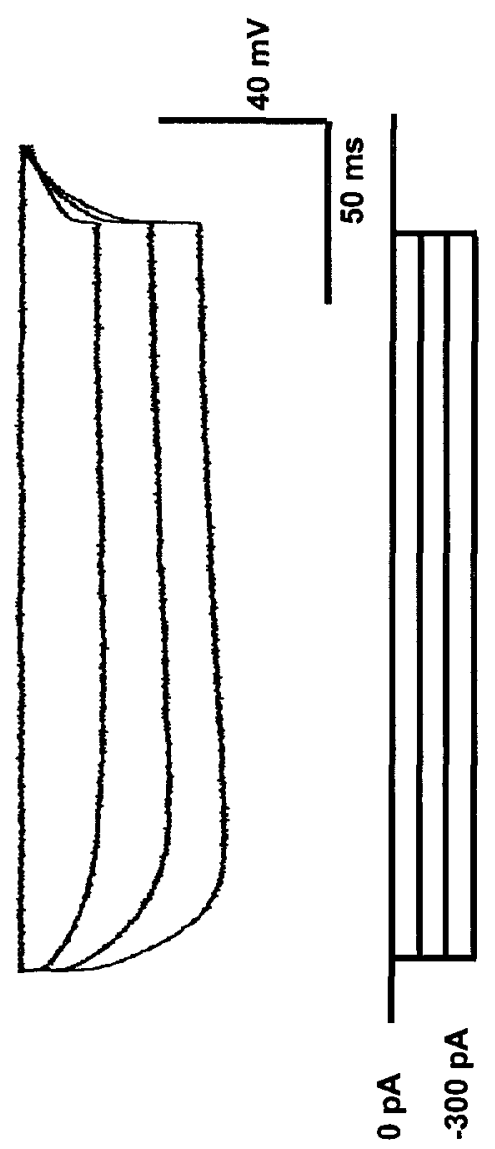


Figure 1 - Effects of salicylate on current-voltage (I-V) relationship. Responses to hyperpolarizing current injection (from bottom to top: $-300,-200,-100 \mathrm{pA}$ ) from an ICC neuron before (A) and during salicylate application (B). (C) The I-V curve for the neuron shown in (A) and (B), before and during drug exposure. (D) The I-V curve for all the neurons before and during salicylate $(n=27)$. 

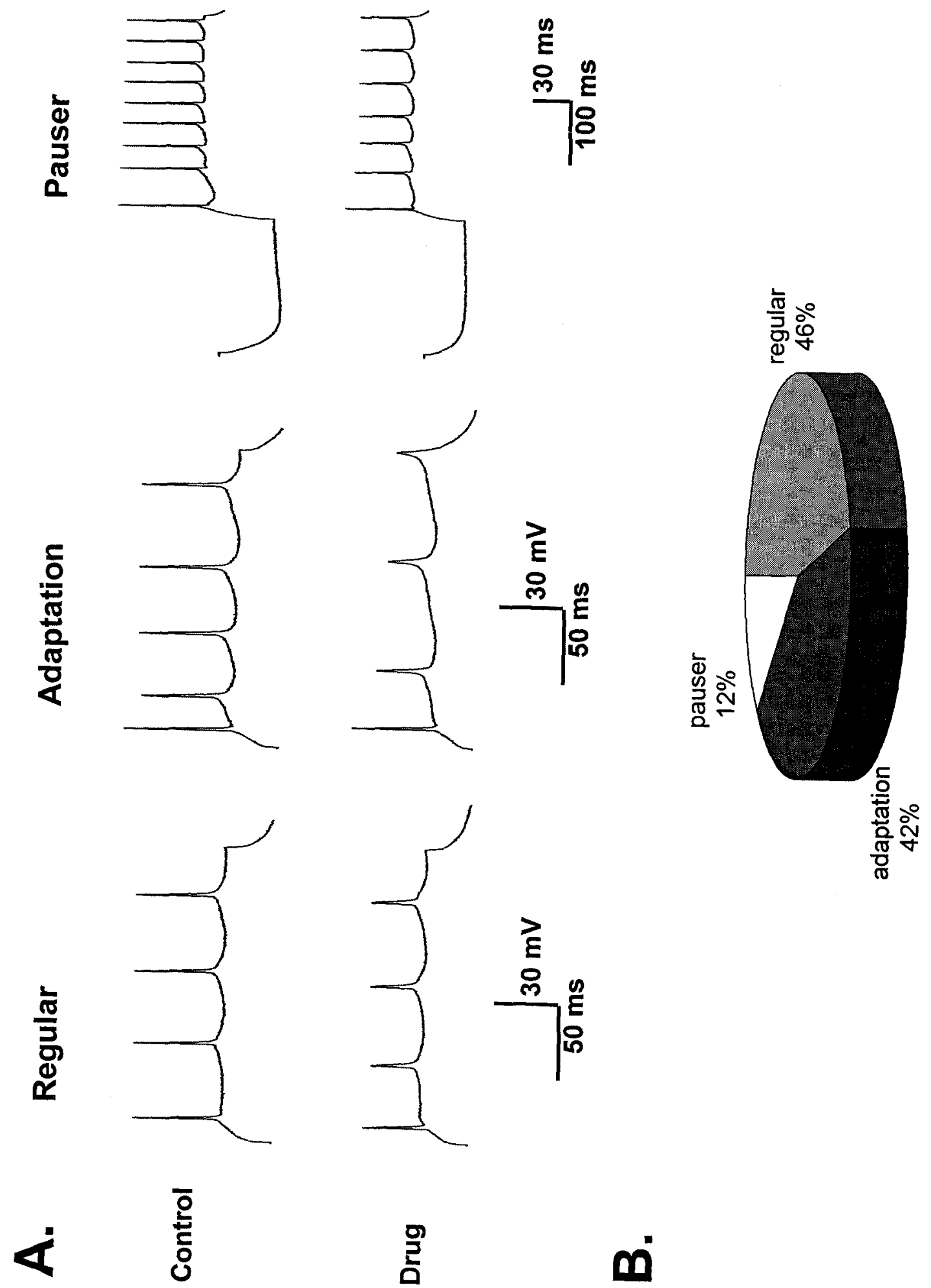
Figure 2 - Effects of salicylate on firing pattern. (A) Responses to a depolarizing current injection in regular-type $(220 \mathrm{pA})$, adaptation-type $(180 \mathrm{pA})$ and pauser-type $(160 \mathrm{pA})$ neurons before and after exposure to salicylate. Note that the cell type was not changed following drug application. (B) Distribution of the ICC neurons based on the firing pattern $(n=26)$. 
A.

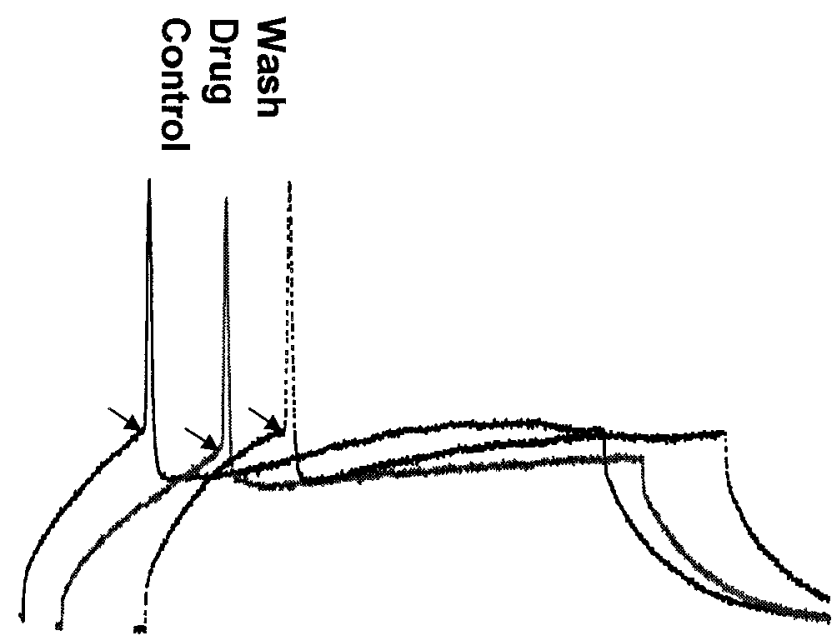

$\int 20 \mathrm{mV}$

$50 \mathrm{~ms}$

B.

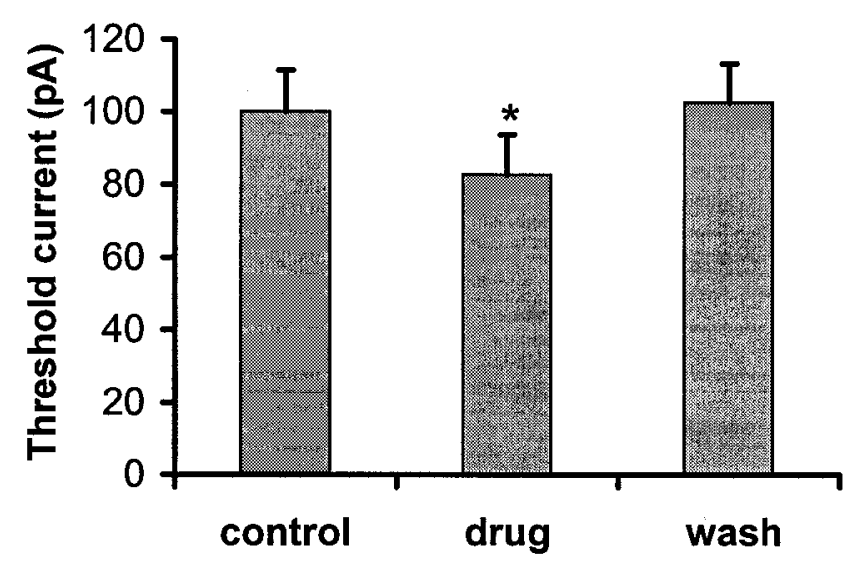

C.

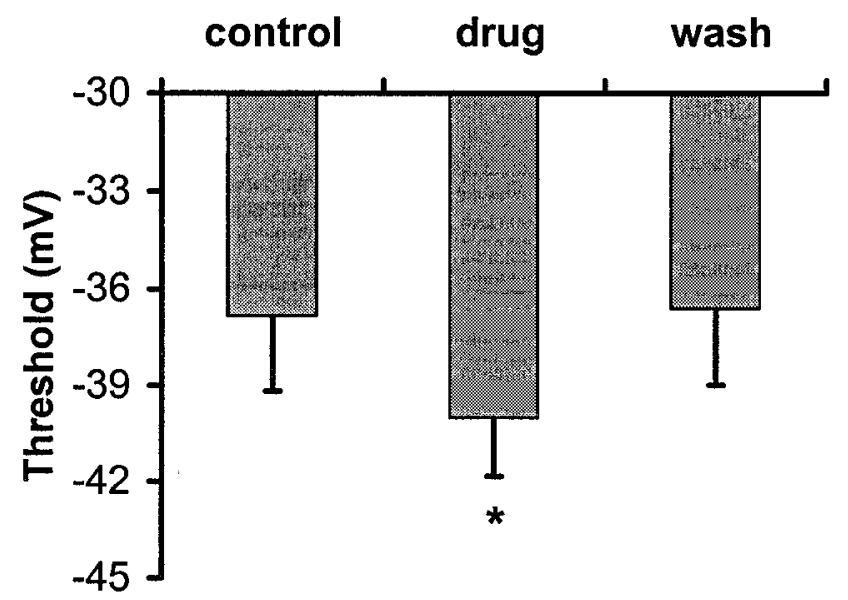


Figure 3 - Comparison of threshold level before and during salicylate exposure and after wash. (A) Responses elicited by depolarizing current injection before drug exposure (140 $\mathrm{pA})$, during drug exposure (120 pA) and after drug wash (140 pA) from an ICC neuron; arrows indicate the threshold of the membrane potential in these three conditions. (B) The mean current threshold in control, drug and wash conditions $(n=15)$. (C) The mean threshold of the membrane potential in these three conditions $(n=15)$. Stars indicate the significant difference. 

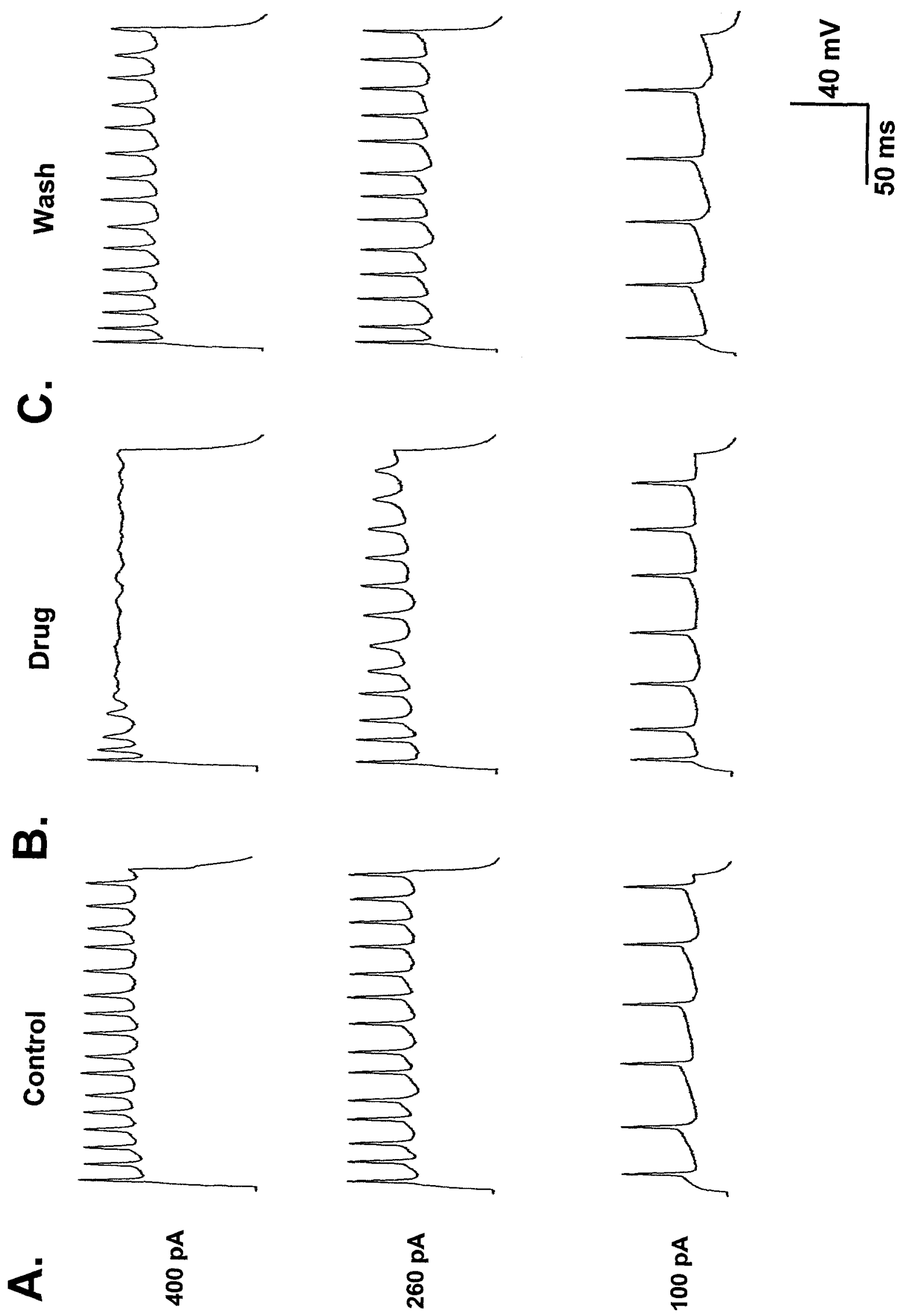
Figure 4 - Effects of salicylate on firing. Responses to depolarizing current injection (from bottom to top: 100, 260, $400 \mathrm{pA}$ ) from an ICC neuron before drug exposure (A), during drug exposure (2 mM) (B), and after wash out the drug (C). Note reductions of firing frequencies during salicylate application compared to those in control condition with increase in depolarizing current injection, which was accompanied by the decreasing in amplitude and widening of APs. These changes were reversible and firing was recovered after wash $(\mathrm{C})$. 
A.

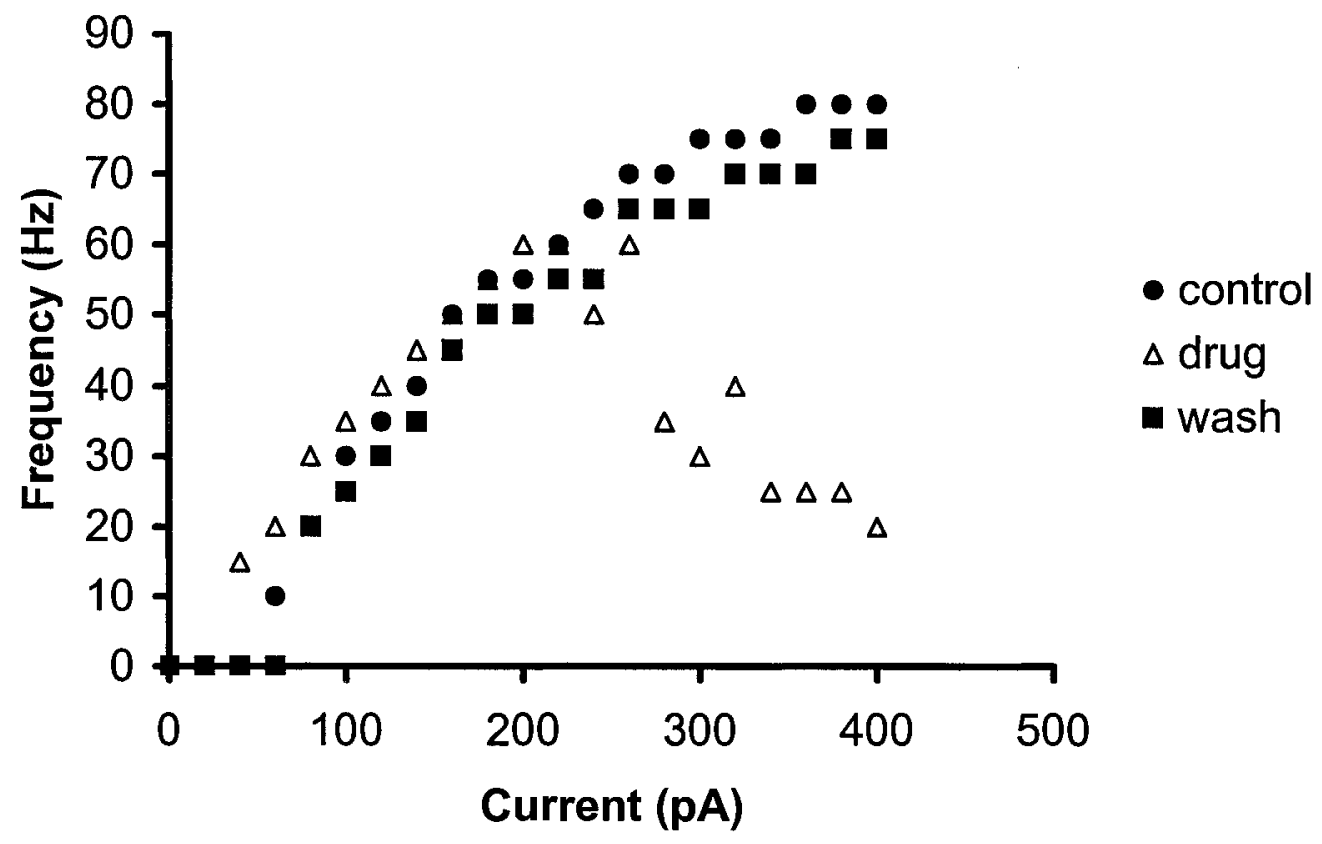

B.

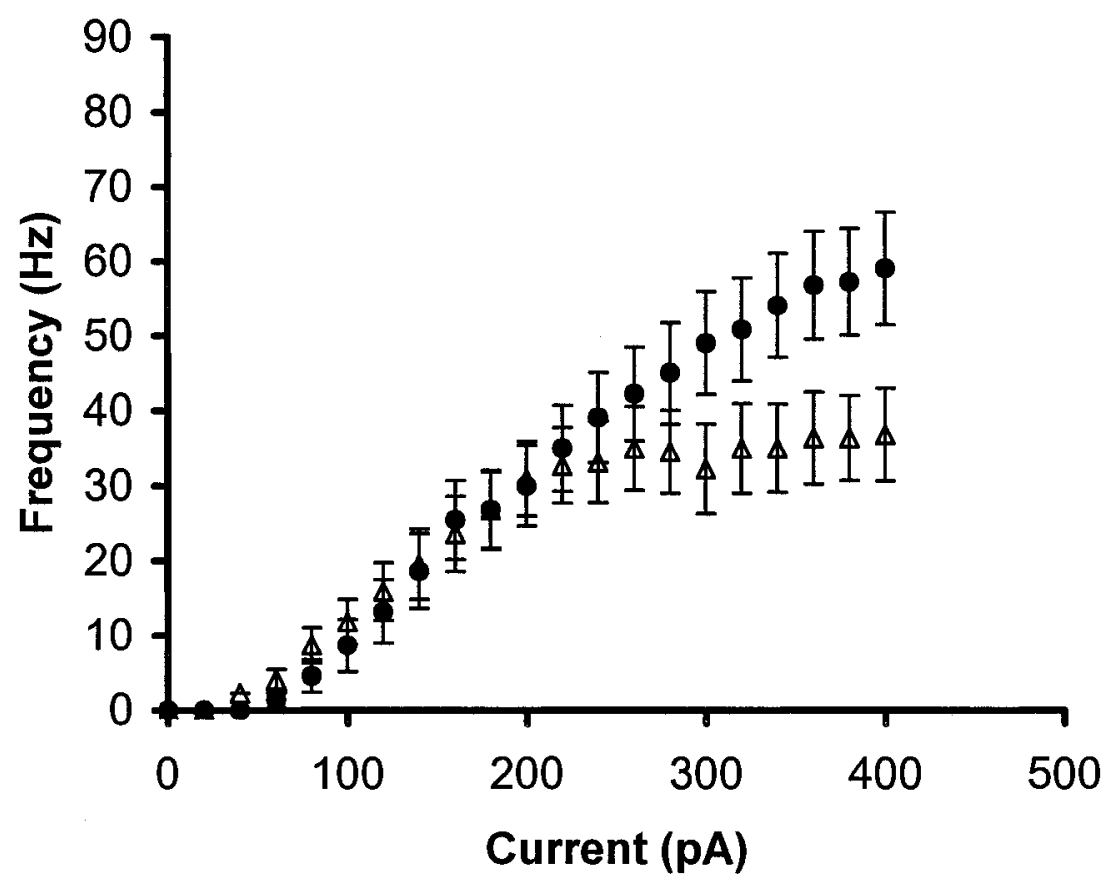

- control

$\Delta$ drug 
Figure 5 - Effects of salicylate on rate level function. (A) The firing frequencies of the ICC neuron shown in Figure 4 were markedly decreased as compared to those in control condition at the levels of current injection of 280 to $400 \mathrm{pA}$. (B) The mean firing frequency of the all ICC neurons tested $(\mathrm{n}=11)$, decreased during salicylate application (1-2 mM) at the levels of 300 to $400 \mathrm{pA}$ as compared to that before the drug. 

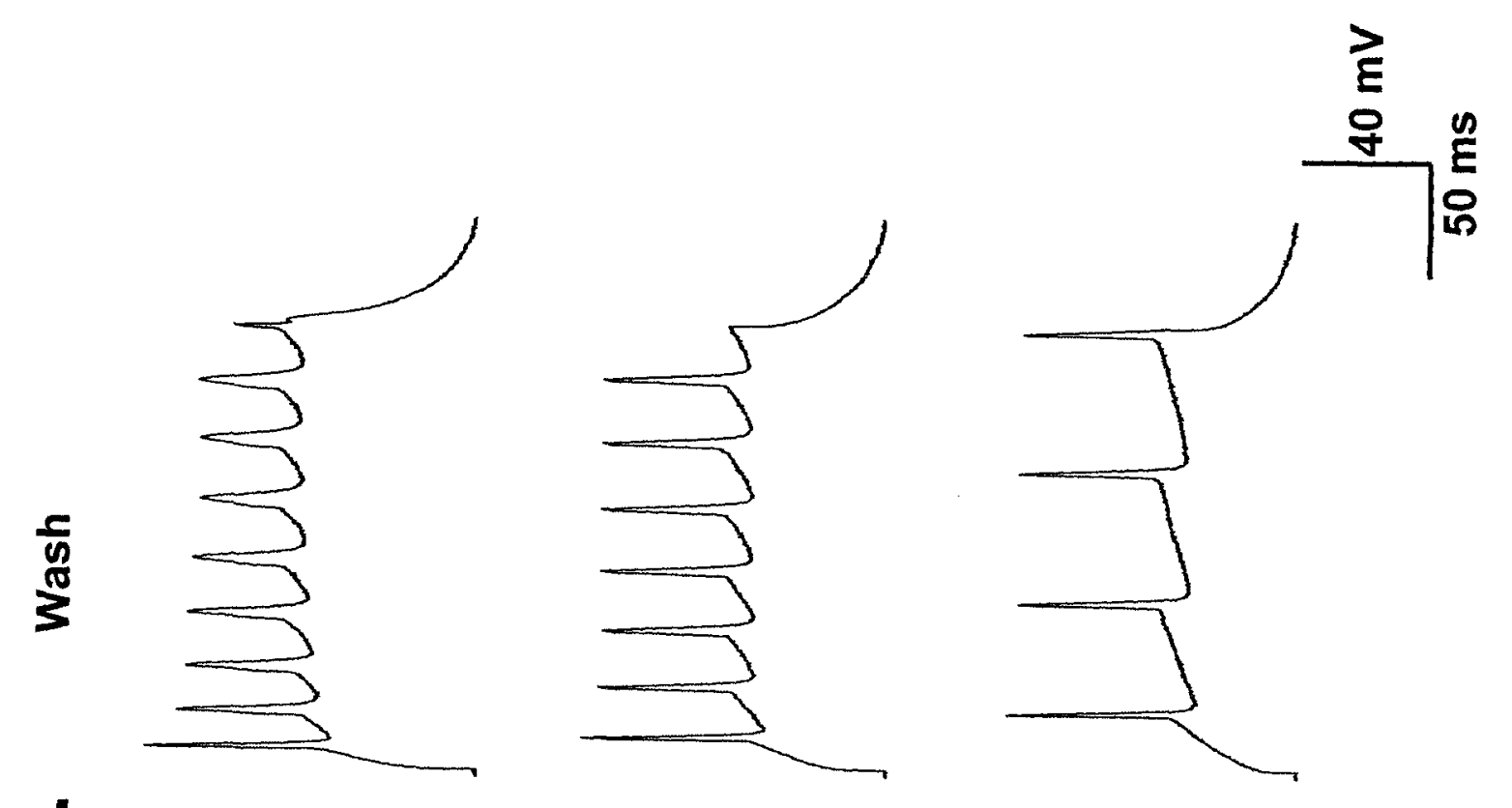

ن
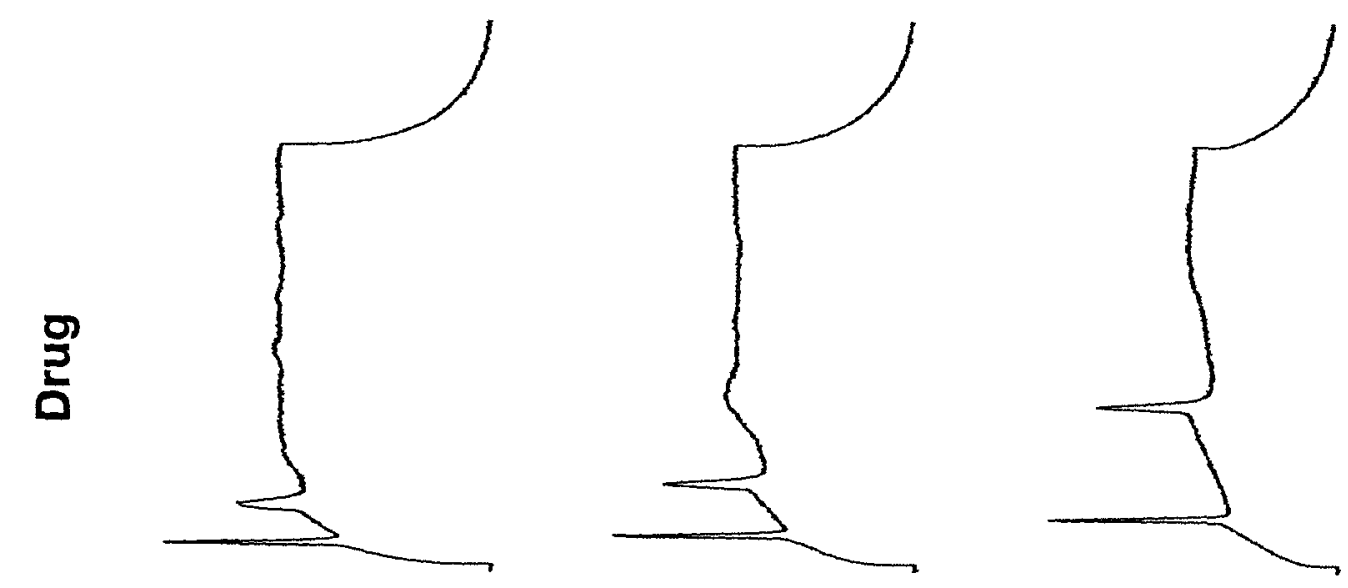

กั
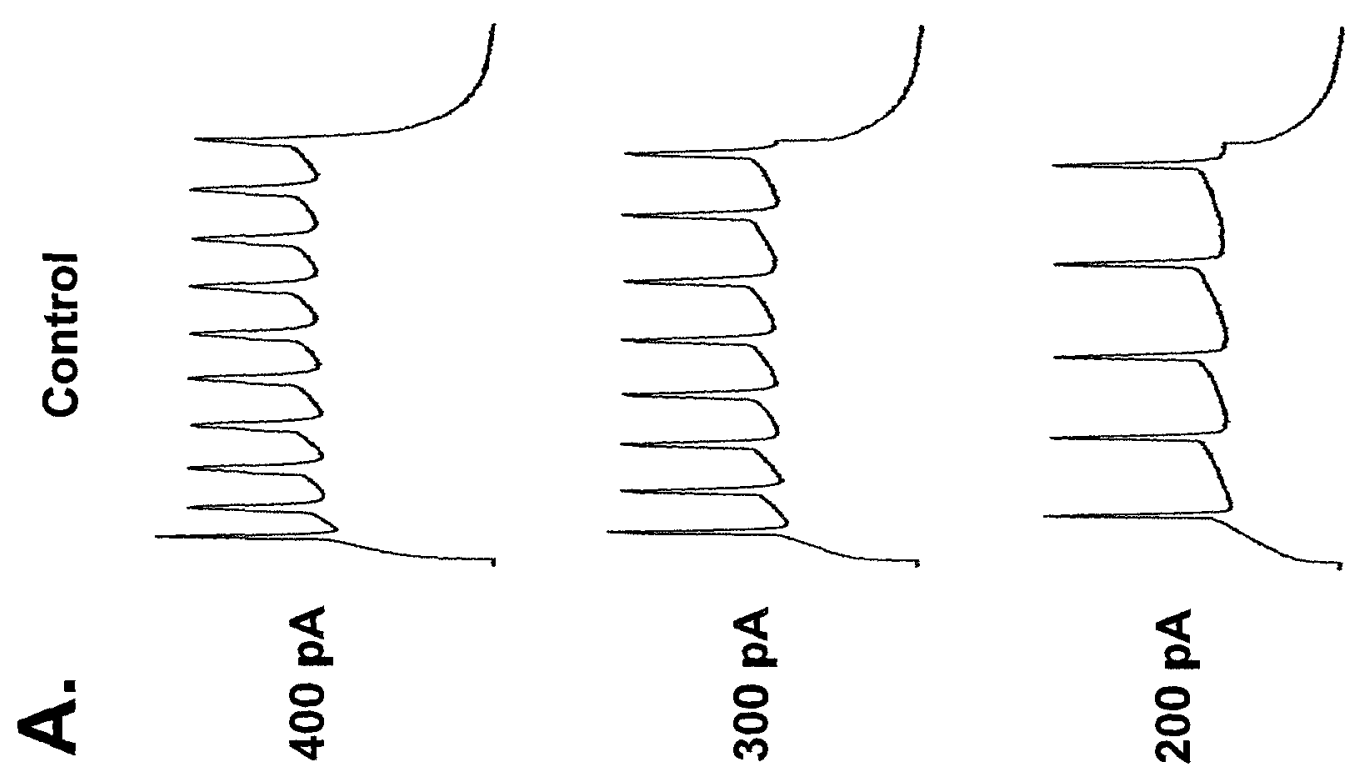
Figure 6 - Effects of salicylate on firing. Responses to depolarizing current injection (from bottom to top: $200,300,400 \mathrm{pA}$ ) from an ICC neuron before drug exposure (A), during drug exposure $(5 \mathrm{mM})(B)$, and after wash $(\mathrm{C})$. Note a prominent reduction of the $\mathrm{AP}$ number and firing frequency, which started at the lower level of current injection $(200 \mathrm{pA})$ as compared with the neuron shown in Figure 4. The firing frequency recovered following wash out the drug(C). 
A.

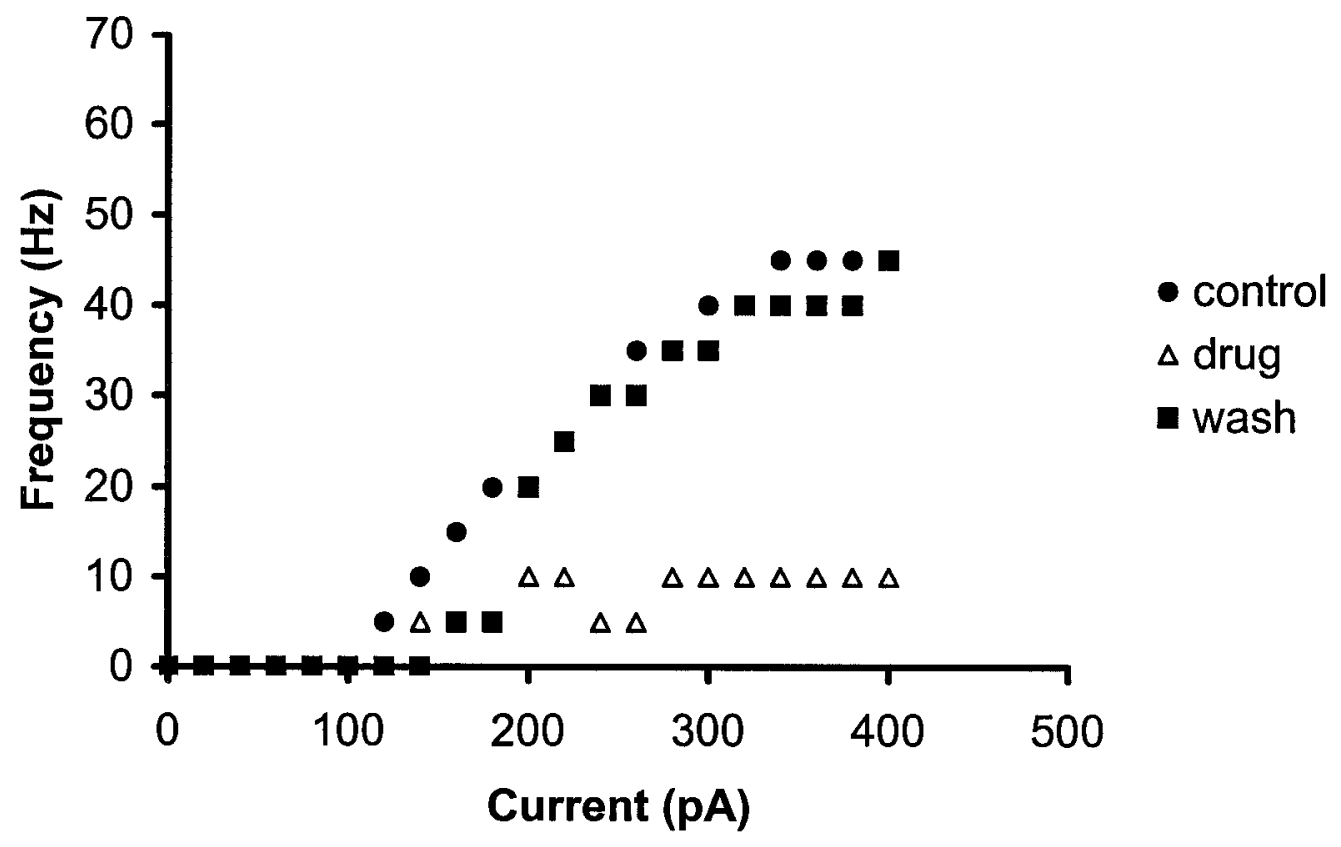

B.

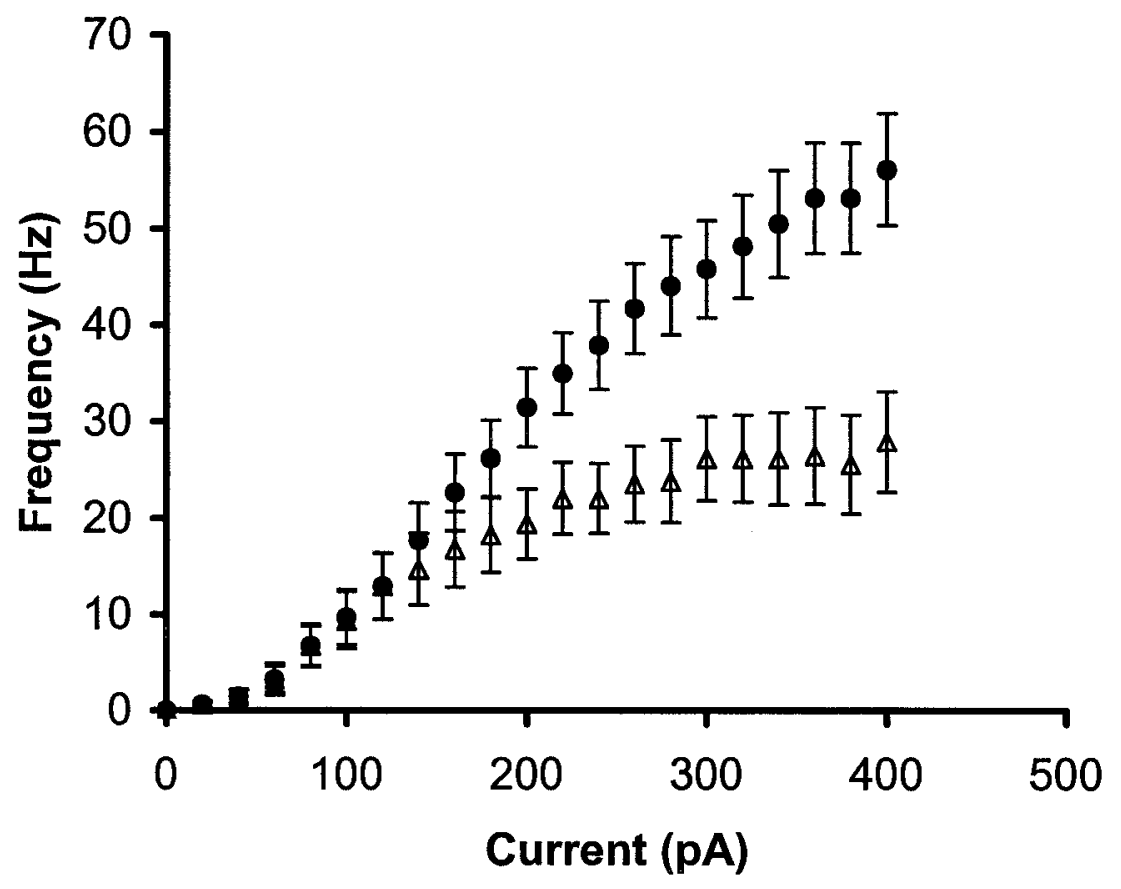

- control

$\Delta$ drug 
Figure 7 - Effects of salicylate on rate level function. (A) The firing frequency of the ICC neuron shown in Figure 6 was greatly reduced at the levels of current injection of 200 to $400 \mathrm{pA}$ during salicylate. (B) The mean firing frequency of the all ICC neurons tested $(n=17)$, decreased during salicylate application $(3-5 \mathrm{mM})$ at the levels of current injection of 200 to $400 \mathrm{pA}$. 


\section{Control}

A.

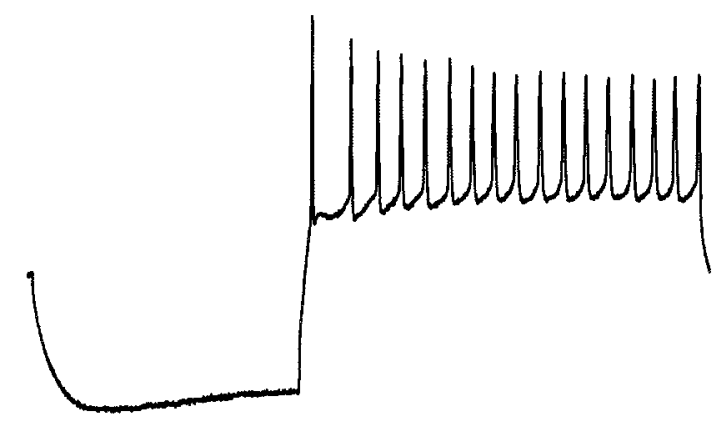

\section{Drug}

B.

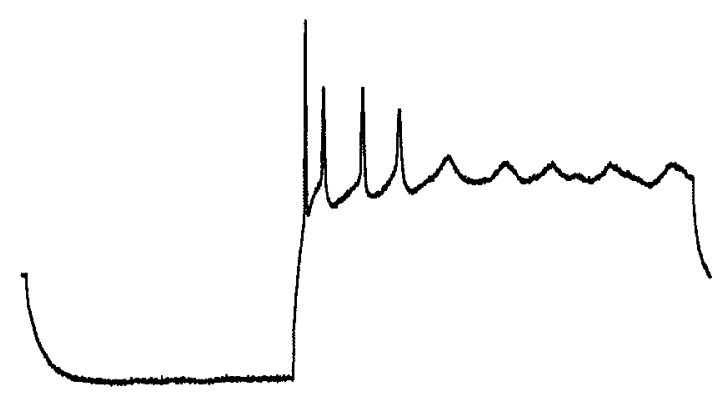

\section{Wash}

C.
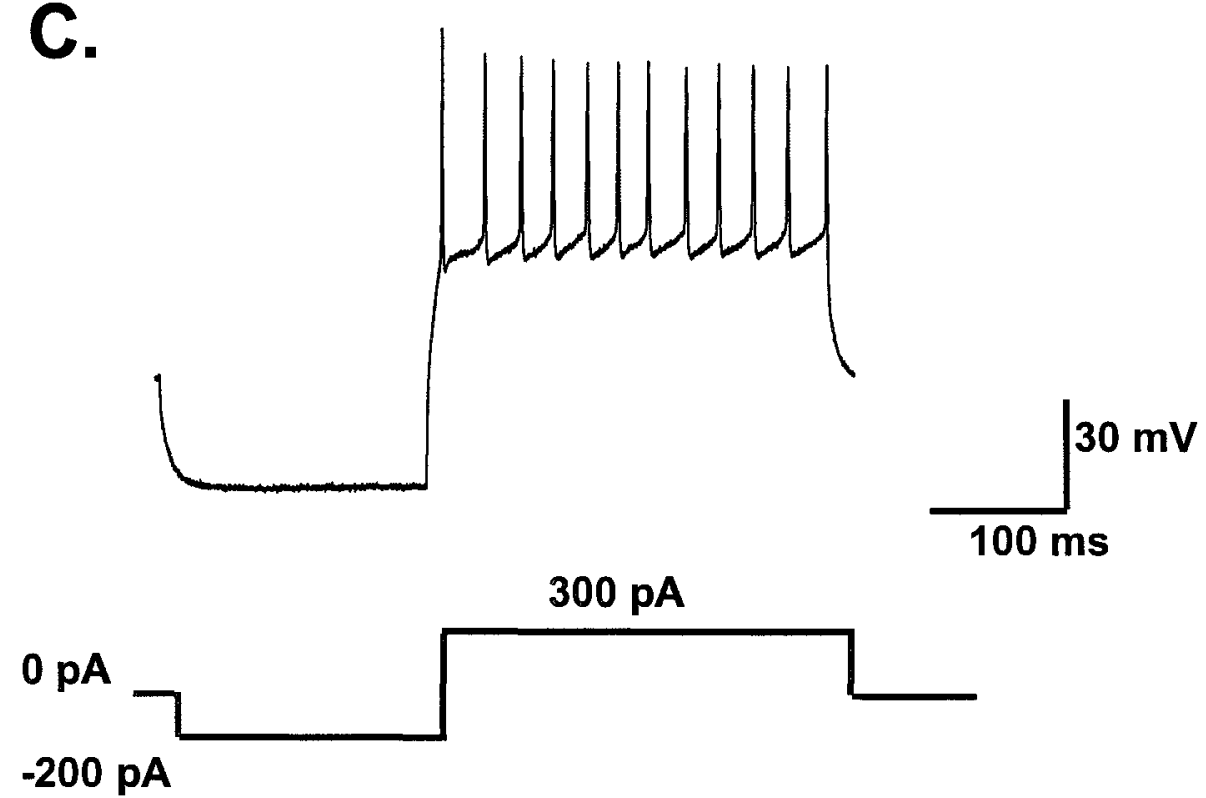
Figure 8 - Effects of salicylate on firing in a pauser neuron. Responses to depolarizing current injection $(300 \mathrm{pA})$ following a fixed prehyperpolarizing current injection $(-200$ pA) before drug exposure (A), during drug exposure (B), and after wash (C). Note the similar alterations in AP properties and firing frequency in this neuron in comparison to the neurons with sustained-regular firing pattern shown in Figures 4 and 6, and the reduction in the pause duration after salicylate application (B). These effects were reversible after wash $(\mathrm{C})$. 


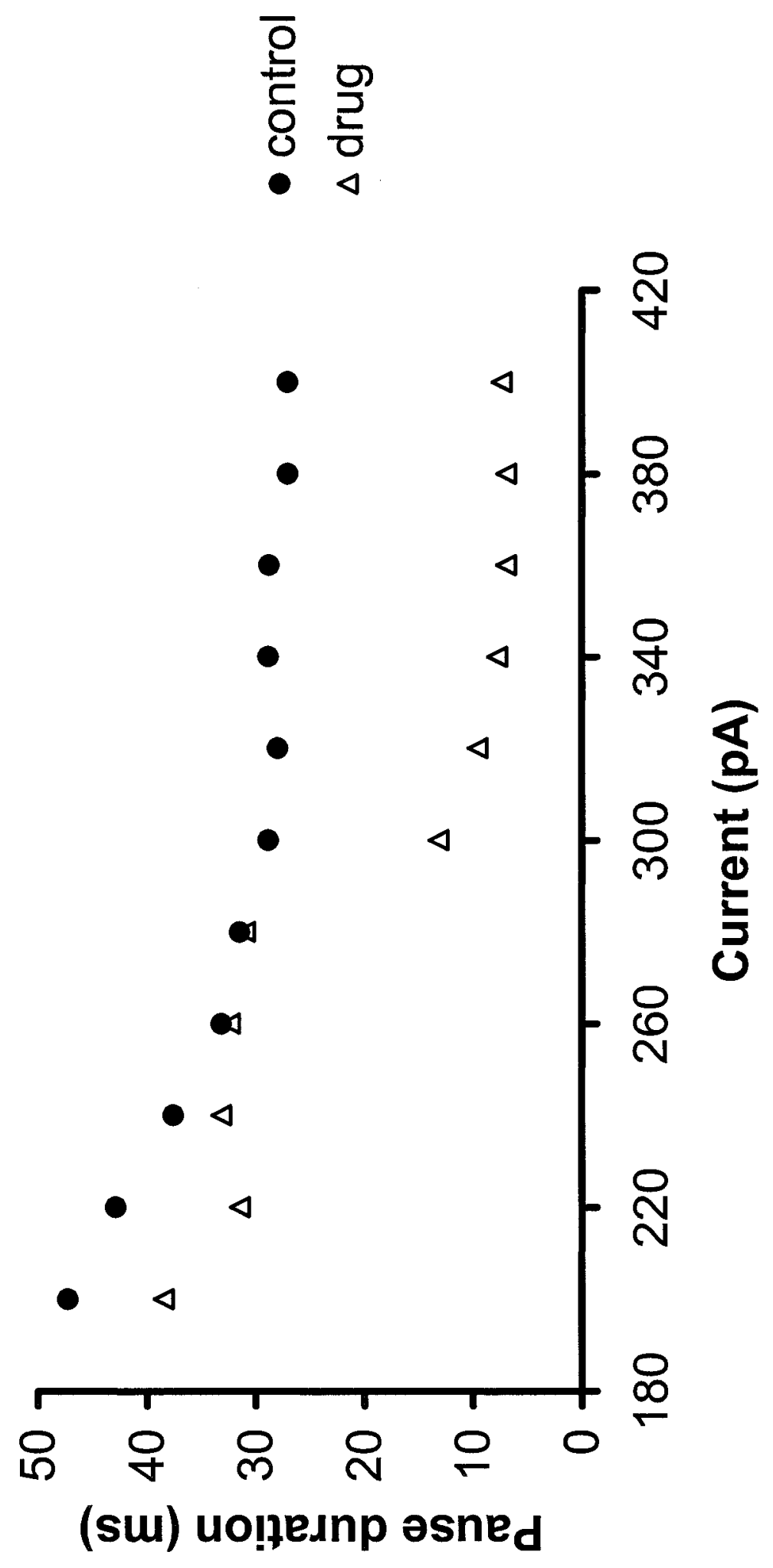


Figure 9 - Effects of salicylate on the pause duration of an ICC neuron. Note the reduction of pause duration by increase in level of depolarizing current injection was more prominent at high levels of depolarizing current injection (300 to $400 \mathrm{pA}$ ) during salicylate application. 
A.
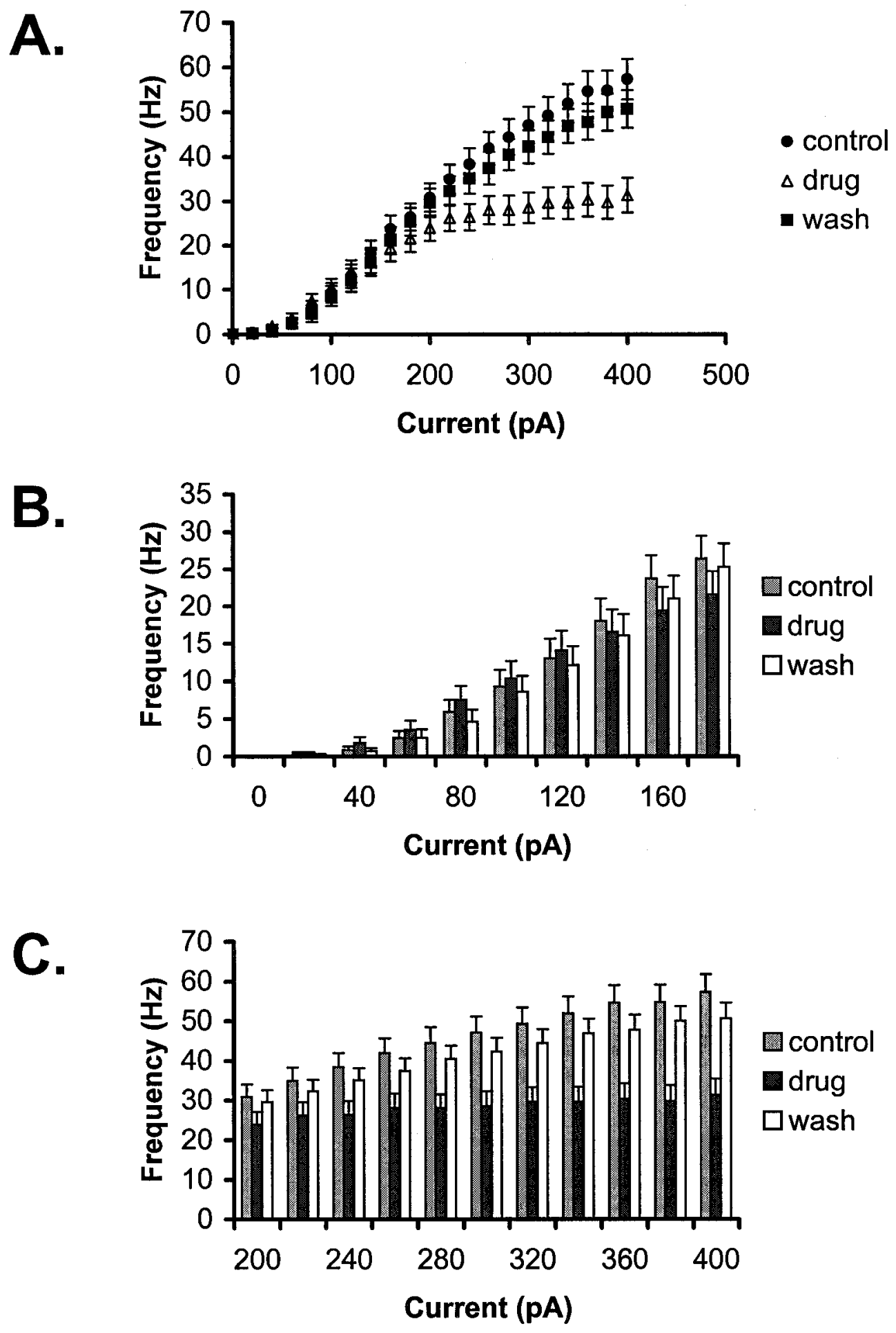
Figure 10 - Effects of salicylate on rate level function. (A) The firing frequency of all the neurons subjected to salicylate application. There was a significant difference in firing frequency between control and drug conditions at the levels of current injection of more than $240 \mathrm{pA}$ to $400 \mathrm{pA}$. (B) The mean firing frequency of the ICC neurons in response to the levels of current injection from 20 to $180 \mathrm{pA}(\mathrm{n}=28)$. The firing frequency was increased by increasing the level of current injection from 20 to $140 \mathrm{pA}$ before and during drug exposure and after wash. (C) The mean firing frequency of the same group of ICc neurons in response to the levels of current injection of 200 to $400 \mathrm{pA}$. Following the increase in the level of current injection from 200 to $400 \mathrm{pA}$, the firing rate progressively increased in control and wash conditions. However, there was a very slight increase in firing frequency during drug exposure. 


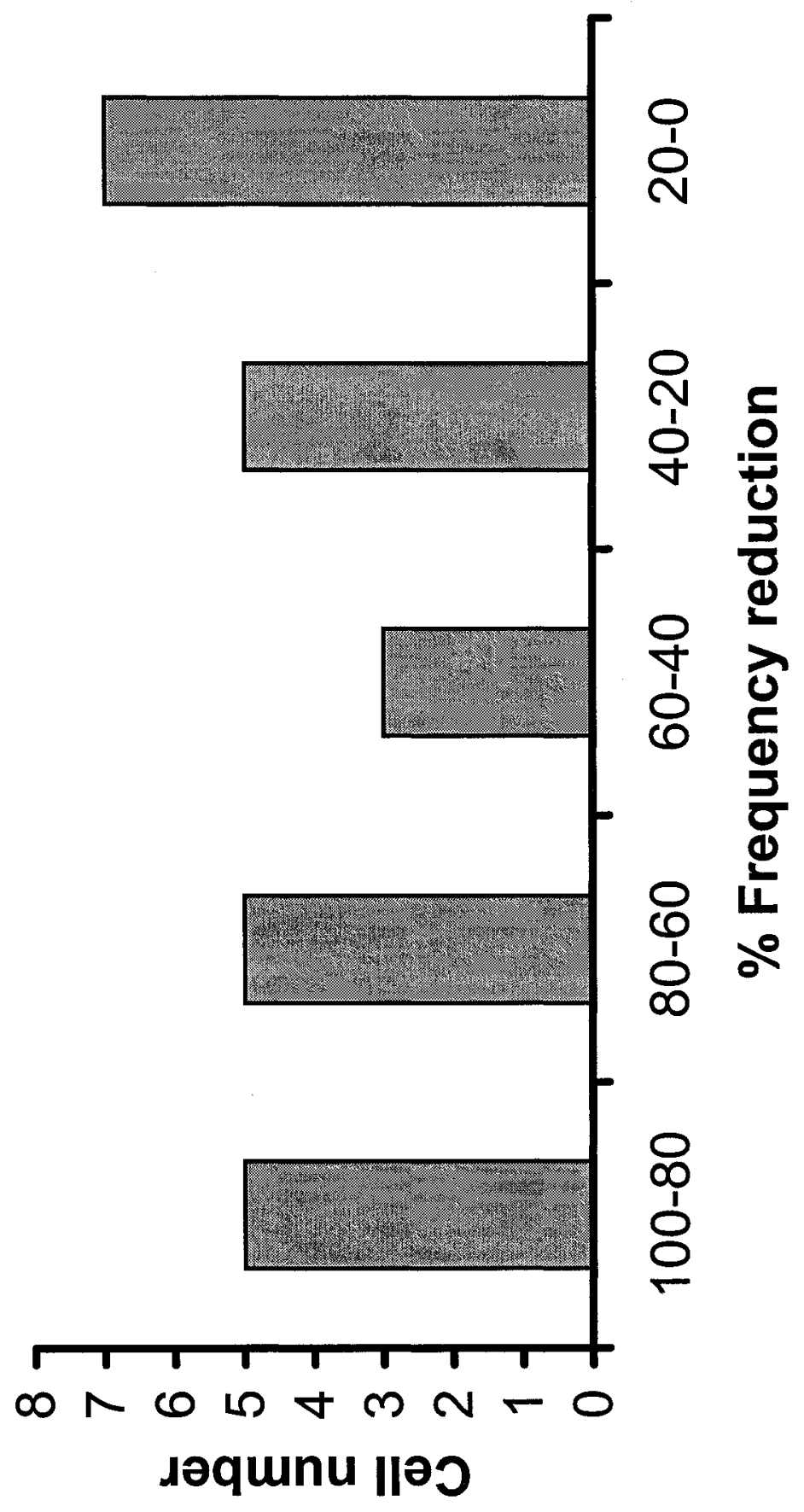

Reproduced with permission of the copyright owner. Further reproduction prohibited without permission. 
Figure 11 - Distribution of sensitivity in firing frequency of ICC neurons to salicylate. Note the frequency reduction was variable among the ICC neurons. 
A.

Control

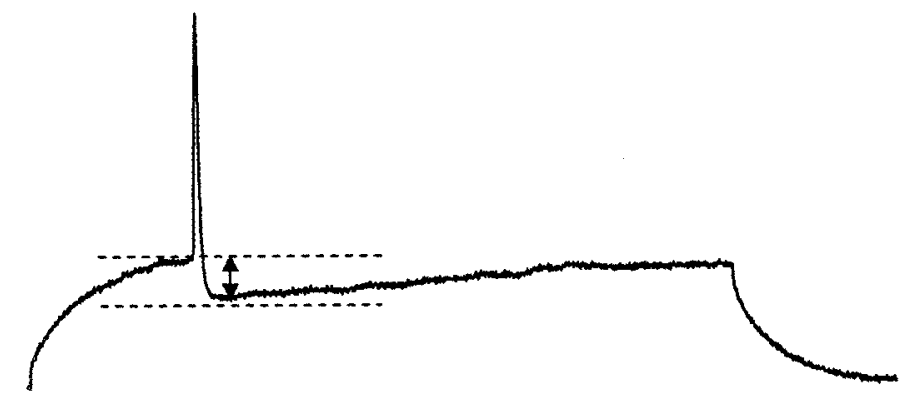

B.

\section{Drug}

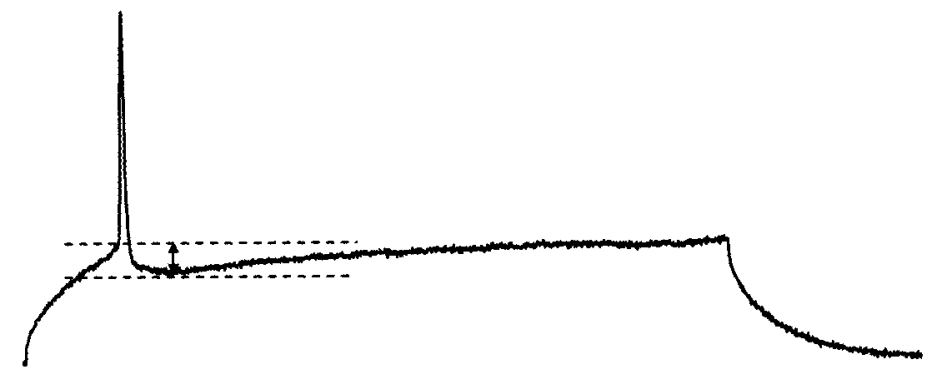

C.

Wash

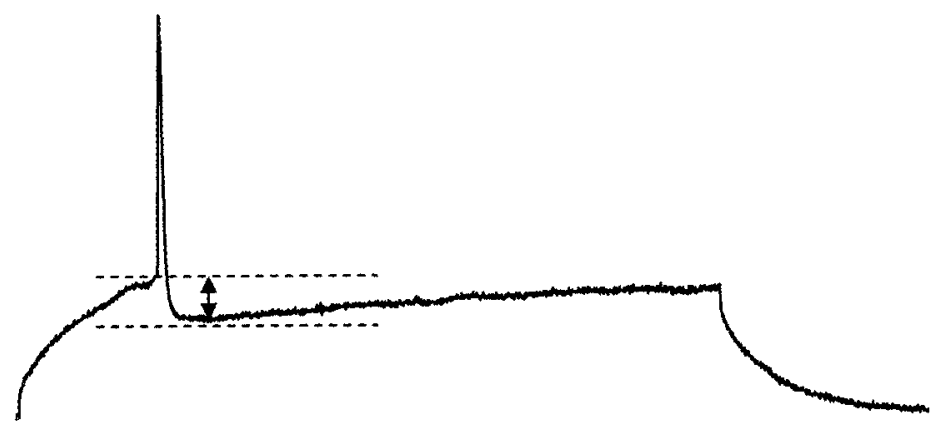

$\frac{}{50 \mathrm{~ms}} 30 \mathrm{mV}$ 
Figure 12 - Effects of salicylate on AHP amplitude. The traces of APs at the threshold level of a neuron before (A), and during drug exposure (B), and after wash (C). The AHP amplitude decreased following salicylate application and recovered after wash. 

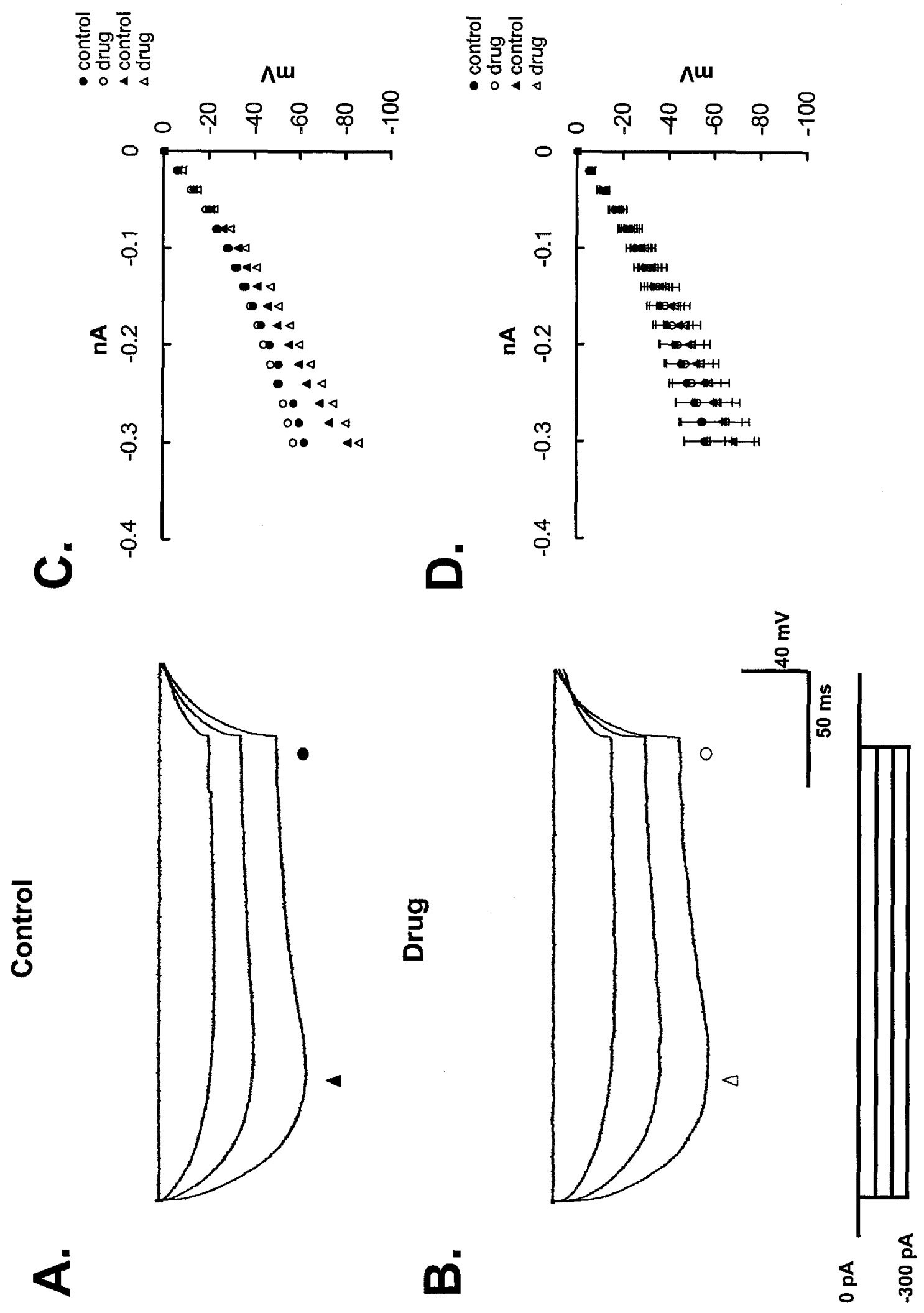
Figure 13 - Effects of salicylate on hyperpolarization-activated current $\left(\mathrm{I}_{h}\right)$. Responses to hyperpolarizing current injection (from bottom to top: $-300,-200,-100 \mathrm{pA}$ ) from an ICC neuron before (A) and during salicylate application (B). (C) The I-V curves for the peak (triangles) and steady state (circles) hyperpolarization shown in (A) and (B) before and during drug exposure. (D) The I-V curves for the peak and steady state hyperpolarization for all the neurons $(n=4)$ with depolarizing sag before and during exposing to salicylate. 


\section{Control}

\section{A.}

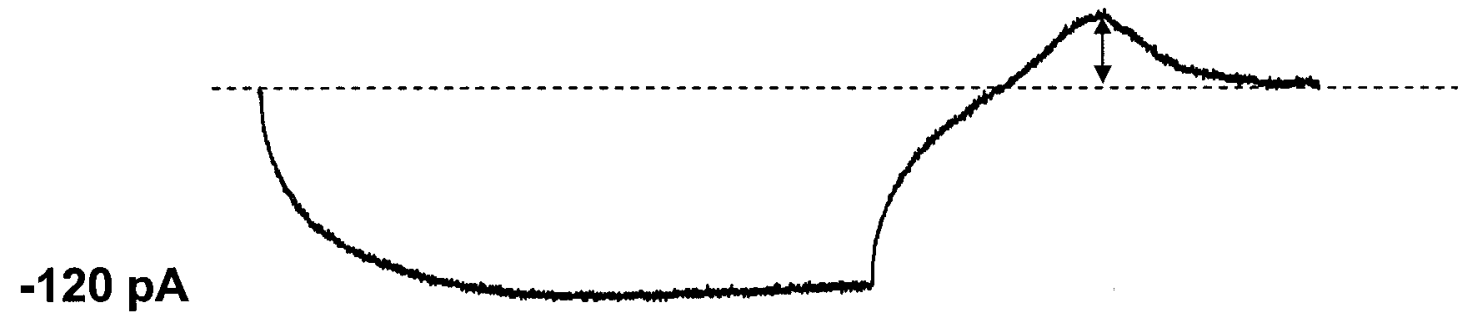

\section{Drug}

B.

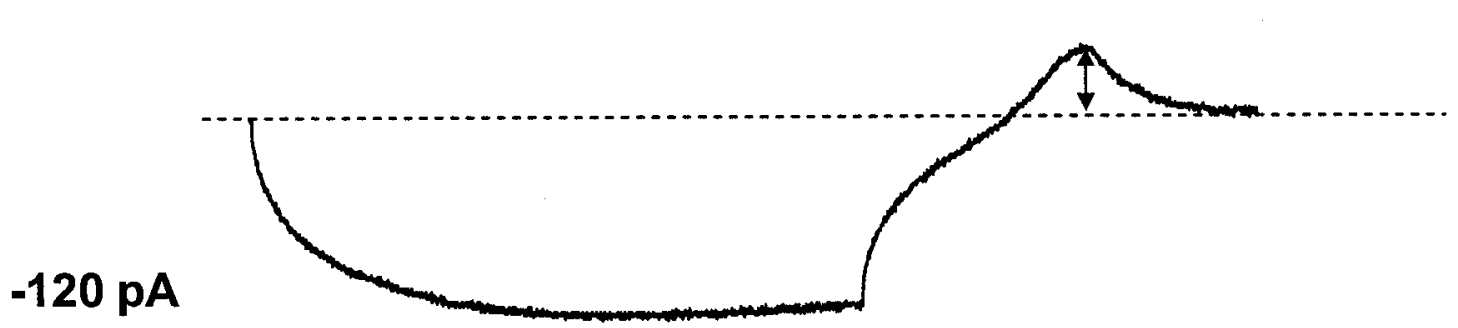

$-120 \mathrm{pA}$

\begin{tabular}{l|l}
$50 \mathrm{~ms}$ & $20 \mathrm{mV}$
\end{tabular} 
Figure 14 - Effects of salicylate on rebound depolarization. Responses to a hyperpolarizing current injection of $-120 \mathrm{pA}$ before (A) and after the drug application (B). Arrows indicate the rebound amplitude that was $13 \mathrm{mV}$ during control condition. Rebound amplitude was not changed $(13 \mathrm{mV})$ at the same level of current injection during salicylate application. 


\section{Discussion}

\subsection{Salicylate and passive membrane properties}

The current-voltage relationship for every ICC neuron tested was approximately linear, and had no obvious change before and during salicylate application. Using the linear portion of the relationship, the slope was used to calculate membrane input resistance. Membrane time constant was derived from the responses to negative current injection. The analysis indicated that the ICC neurons exposed to salicylate displayed no significant difference in the input resistance and time constant compared to when the neurons were in control condition. The results of the current study are comparable with those in a study of sodium salicylate on inhibitory postsynaptic currents in neurons of rat auditory cortex (Wang et al, 2006). This study revealed no change in the input resistance of neurons following salicylate application The results of the current study that salicylate did not change resting membrane potential, input resistance and time constant suggest that salicylate does not affect passive membrane properties of ICC neurons.

\subsection{Salicylate and membrane excitability}

\subsubsection{Firing pattern}

The ICC neurons encountered in this study expressed different firing patterns classified as regular-type, adaptation-type, and pauser-type in response to depolarizing and hyperpolarizing current injections. The regular type neurons were the most commonly encountered firing pattern constituting $46 \%$ of the population, followed by adaptation-type (42\%) and pauser-type (12\%). The regular-type cells displayed a firing pattern in which the interspike intervals were quite regular, unlike the adaptation and 
pauser-type cells, which showed different interspike intervals throughout the depolarizing current pulse. In adaptation-type cells, the earlier interspike intervals were shorter than the later intervals. The pauser-type cells displayed a pronounced pause after the first spike. This relatively long interspike period was followed by more regular firing.

Analysis of salicylate effects on the firing patterns of ICC neurons in the current study showed that salicylate did not change the firing pattern of the cells. According to Sivamakrishnan and Oliver's study (2001), each of these firing patterns is associated with specific types of $\mathrm{K}^{+}$current. The regular-type pattern is attributable predominantly to 4AP-sensitive delayed rectifying $\mathrm{K}^{+}$channels. The sustained firing cells that adapt during a current injection have, in addition to delayed rectifying $\mathrm{K}^{+}$currents, apamin-sensitive $\mathrm{K}^{+}$currents that are likely to be carried through $\mathrm{Ca}^{2+}$-dependent $\mathrm{K}^{+}$channels. The pausertype cells have an A-current through A-type $\mathrm{K}^{+}$channels, which is responsible for the pause. Following salicylate application, there was no change in firing pattern of the representative pauser-type cell (Fig. 8). All of the above results suggest that salicylate has little effect on activity of delayed rectifier, $\mathrm{Ca}^{2+}$-activated and A-type $\mathrm{K}^{+}$channels at low levels of depolarization, and these channels are still active enough to contribute to the distinct firing patterns. However, at higher levels of depolarizing current injection, the distinct firing pattern could not be identified, because the number of spikes greatly decreased (see discussion in later section).

\subsubsection{Firing abilities}

\subsubsection{Threshold of firing}

The result of this study revealed that following salicylate application, ICC neurons required a lower level of current injection to initiate firing as compared to control 
condition. The reduction in the current threshold during the drug exposure may be related to the effect of salicylate on voltage-gated $\mathrm{Na}^{+}$and $\mathrm{K}^{+}$channels. A study by $\mathrm{Liu}$ and $\mathrm{Li}$ (2004) indicated that salicylate blocked sodium channels and decreased the amplitudes of $\mathrm{I}_{\mathrm{Na}}$, without changing the voltage dependence of activation of sodium channels in IC neurons. Liu and Li's study (2004) also indicated that salicylate molecules have a higher affinity for the inactivation state than for the resting state of the channels. In addition, these authors reported (2004) that salicylate inhibited the current amplitude of the delayed rectifier potassium channels $\left(\mathrm{I}_{\mathrm{K}(\mathrm{DR})}\right)$. Action potential threshold is the specific value of membrane potential at which the net ionic current (the sum of inward sodium current and outward potassium current) just changes from outward to inward, depositing a net positive charge on the inside of the membrane capacitance (Koester and Siegelbaum, 2000). Although salicylate suppresses both the inward sodium and outward potassium currents, the relative extent of blockade on $\mathrm{Na}^{+}$vs. $\mathrm{K}^{+}$by salicylate is not clear. In addition, salicylate has a less affinity for resting state than inactivation state of the sodium channels ( $\mathrm{Liu}$ and $\mathrm{Li}, 2004$ ). Therefore, the lower threshold of AP during salicylate application may be attributed to blockade of $\mathrm{K}^{+}$conductance to a greater extend relative to $\mathrm{Na}^{+}$conductance by salicylate at membrane potential near the threshold.

\subsubsection{Firing frequency}

The result of this study revealed that salicylate decreased the firing rate of ICC neurons during drug application at higher levels of depolarizing current injection. There was a significant decrease in firing frequency following salicylate exposure in comparison to control and wash conditions at levels of 200 to $400 \mathrm{pA}$ of current injection. In addition to decrease in spike number, the APs became wider and smaller during drug, 
which was more obvious as more depolarizing current was injected. A few APs at the beginning of the current pulse were seen, and these spikes had smaller amplitudes and longer durations than APs in the control condition. These smaller spikes (during drug exposure) were followed by several humps with even smaller amplitude and longer duration. It was also observed that there was a tendency for these changes to occur at lower levels of depolarizing current injection with increasing in salicylate dose, i.e., the firing frequency of ICC neurons that received 1-2 mM salicylate, decreased at the levels of current injection of $300 \mathrm{pA}$ to $400 \mathrm{pA}$, but those exposed to 3-5 mM salicylate, showed firing frequency reduction at the levels of $200 \mathrm{pA}$ to $400 \mathrm{pA}$. Therefore, it seems that when the salicylate dose increases, the firing frequency reduces more.

The effect of salicylate on firing frequency is probably due to block of the voltage-gated sodium and potassium channels. According to Liu and Li's study (2004), salicylate had effect on the inactivation state of voltage-gated sodium channels, and shifted the voltage dependence of inactivation to more hyperpolarizing direction. Salicylate also delayed the recovery from the inactivation state of the sodium channel. Their results can explain the reduction in firing frequency and AP amplitude of the neurons in the current study. This means that the sodium channels needed more time to come back to their resting state, and get ready to fire next AP during depolarization.

Li et al. study (1998), demonstrated that substitution of a reduced-sodium solution for the normal ACSF reduced number of spikes throughout the current pulse. Also in the current study, the number of spikes was reduced during the current pulse after salicylate application, which not only confirms the role of sodium channels in repetitive firing, but 
also suggests that salicylate decreases firing frequency through blocking of sodium channels.

The transient outward potassium channel and the delayed rectifier potassium channel are two main types of potassium channels, which have been identified in IC neurons by their activation and inactivation kinetics and pharmacological sensitivities (Sivamakrishnan and Oliver, 2001). Any change in the properties of these potassium channels can directly affect neuronal activity such as repolarization, firing rate and the shape of the action potential. Liu and $\mathrm{Li}$ (2004) revealed that salicylate blocked voltagegated potassium channels, and specifically decreased the current amplitude of $I_{\mathrm{K}(\mathrm{A})}$ and $I_{\mathrm{K}(\mathrm{DR})}$. This blocking effect was much stronger on $I_{\mathrm{K}(\mathrm{DR})}$ than on $I_{\mathrm{K}(\mathrm{A})}$. Salicylate significantly affected activation and inactivation kinetics of $I_{\mathrm{K}(\mathrm{DR})}$, including shifting the steady-state activation and inactivation curves in a more hyperpolarizing direction. Since $I_{\mathrm{K}(\mathrm{A})}$ is transient, the repolarization mainly depends on activation of $I_{\mathrm{K}(\mathrm{DR})}$. Therefore, blockade of $I_{\mathrm{K}(\mathrm{DR})}$ would retard repolarization after rising phase of the AP. The decrease in spike number and change in AP shape (longer duration) by salicylate during depolarizing current pulse at higher current levels is very likely due to the blocking effect on $I_{\mathrm{K}(\mathrm{DR})}$. Sivaramakrishnan and Oliver's study (2001) indicated that the duration of an AP of ICC neurons became longer with application of the $I_{\mathrm{K}(\mathrm{DR})}$ antagonist, 4-AP, which suggests that longer duration of AP during salicylate application in the current study is due to the blocking effect of salicylate on $I_{\mathrm{K}(\mathrm{DR})}$.

The alterations in the firing frequency and AP properties of ICC neurons by salicylate at higher current levels in the current study are also possibly due to a combination of blocking of sodium and potassium channels. The ionic basis of the APs in 
rat ICX neurons was verified by Ahuja and $\mathrm{Wu}$ (2007) using pharmacological manipulations. The classical sodium and potassium conductance were abolished using $\mathrm{Na}^{+}$and $\mathrm{K}^{+}$channel blockers, TTX (tetradotoxin) and TEA respectively, and typical spikes that mediated by $\mathrm{Na}^{+}$and $\mathrm{K}^{+}$currents were totally blocked. Therefore, reduction of amplitude and duration of AP and spikes number at higher current levels suggests that salicylate suppresses both $\mathrm{Na}^{+}$and $\mathrm{K}^{+}$currents through $\mathrm{Na}^{+}$and $\mathrm{K}^{+}$channels. These suppression effects of salicylate are not as potent as TTX and TEA that completely block APs at all levels of depolarizing current injections, because at lower levels of depolarizing current pulses, regular spikes are still present and at higher levels, a few early spikes were also typical spikes. Therefore, at lower levels of depolarizing current injections there are probably enough $\mathrm{Na}^{+}$and $\mathrm{K}^{+}$currents through $\mathrm{Na}^{+}$and $\mathrm{K}^{+}$channels to elicit spikes whose shape and number are similar to those in control condition. However, at higher levels of depolarizing current, the neurons require more $\mathrm{Na}^{+}$and $\mathrm{K}^{+}$currents to elicit more APs, but suppression effects on $\mathrm{Na}^{+}$and $\mathrm{K}^{+}$currents by salicylate results in great reductions in spikes number and changes in shape of APs.

The effect of salicylate on a pauser-type neuron revealed the reduction in the first interspike interval (pause) in the current study. In Perruzzi et al.'s (2000) study a small fraction of sustained cells were characterized by a pause in their firing when a depolarizing current injection was preceded by a hyperpolarizing current step. The pause firing pattern is caused by removal of inactivation of an A-type $\mathrm{K}^{+}$channel by prehyperpolarization and its subsequent activation by depolarization (Kanold and Manis, 1999). This fast transient $\mathrm{K}^{+}$conductance from activated A-type $\mathrm{K}^{+}$channels can be observed when the cell is depolarized following a prehyperpolarization 
(Sivaramakrishnan and Oliver, 2001). The pauser neurons encountered in this study initially appeared to be regular-type cells. However, when a prehyperpolarization step was employed, they revealed a pauser-type firing pattern. After launching prehyperpolarizing current injection, the inactivation of the A-type channels was removed, and then depolarization further activated the faster transient A-type $\mathrm{K}^{+}$current.

Liu and Li (2004) showed that salicylate blocked A-type potassium channels and decreased the amplitude of $I_{\mathrm{K}(\mathrm{A})}$ without affecting its activation and inactivation kinetics, which can explain why salicylate decreased the duration of the pause in the current study.

\subsection{Salicylate and action potentials properties}

\subsubsection{Action potential}

Analysis of action potentials at the threshold levels of ICC neurons did not show any change in amplitude and duration following salicylate application. This result suggests that although salicylate suppresses the activity of $\mathrm{Na}^{+}$and $\mathrm{K}^{+}$channels around the threshold level, the channels are still active enough to generate one normal AP during application of salicylate.

\subsubsection{Afterhyperpolarization}

Afterhyperpolarization (AHP) or undershoot is a transient hyperpolarization that follows an action potential, and occurs because the permeability of the membrane to $\mathrm{K}^{+}$is greater than during the resting state. Analysis of the AHP following salicylate application in the current study indicated a decrease in amplitude, without any change in half-width. The ionic basis of the AHP in ICX neurons was determined in Ahuja and Wu's study (2007), by manipulation of $\mathrm{Ca}^{2+}$ concentration in the ACSF. Their observations suggest 
that the undershoot in some ICX neurons are mediated by two different types of $\mathrm{K}^{+}$ channels, the classical voltage-dependent $\mathrm{K}^{+}$channels, and $\mathrm{Ca}^{2+}$-activated- $\mathrm{K}^{+}$channels. Other studies also reveal that AHP is attributed to $\mathrm{Ca}^{2+}$ - activated $\mathrm{K}^{+}$currents because it was abolished by charybdotoxin (a specific blocker of calcium-activated potassium channels) and its amplitude was decreased by removal of extracellular $\mathrm{Ca}^{2+}$ (Smith 1992; Sivaramakrishnan and Oliver 2001).

Salicylate reduced the AHP amplitude of the ICC neurons in the current study, which suggests that salicylate not only blocks the currents through delayed-rectifier $\mathrm{K}^{+}$ channels, but also $\mathrm{Ca}^{2+}$-activated- $\mathrm{K}^{+}$channels. Further study is required to identify which type of $\mathrm{Ca}^{2+}$-activated- $\mathrm{K}^{+}$channels is affected by salicylate.

\subsubsection{Hyperpolarization-activated current $\left(I_{h}\right)$}

Hyperpolarization-activated current $\left(I_{h}\right)$ is a mixed-cation current that is carried out through a type of channel that opens at negative potentials. These channels are mixed cation-channels that are permeable to $\mathrm{Na}^{+}$and $\mathrm{K}^{+}$. The effect of these channels is to initiate slow depolarization if the membrane potential becomes very negative. $I_{h}$ is generally activated at levels of hyperpolarization more negative than the cell's resting potential and its magnitude increases as the level of hyperpolarization increases. Therefore, $I_{h}$ may act as a safety mechanism, which is activated when the cell becomes increasingly hyperpolarized and helps depolarize the cell to normal working conditions.

Some ICC neurons encountered in the current study revealed the existence of $I_{h}$ as there was a depolarizing sag (representing a hyperpolarizing-activated current) following an initial hyperpolarization during hyperpolarizing current injections (Koch and Grothe, 2003). The data did not show any change in magnitude of the depolarizing sag following 
salicylate application in comparison to control condition, which suggests that salicylate does not have any effect on hyperpolarization-activated current $\left(I_{h}\right)$.

\subsubsection{Rebound after hyperpolarization}

When hyperpolarizing current steps were applied to the ICC neurons, some neurons displayed a broad rebound depolarization following membrane hyperpolarization. Several studies (Sivaramkrishnan and Oliver, 2001; Smith, 1992; Ahuja and $\mathrm{Wu}, 2007$ ) have indicated the ionic basis of the rebound depolarization after hyperpolarization. The rebound depolarization was blocked when the cells were bathed in $0 \mathrm{Ca}^{2+} \mathrm{ACSF}$, suggesting that it was attributable to currents flowing through calcium channels.

Following salicylate application on ICC neurons with a rebound depolarization, no obvious effect on rebound amplitude was seen in current study, which suggests that salicylate does not have any effect on the low threshold $\mathrm{T}$-type $\mathrm{Ca}^{2+}$ current that is responsible for rebound depolarization after hyperpolarization (Russier et al., 2003; Sivaramakrishnan and Oliver, 2006).

\subsection{Effects of salicylate on ICC neurons and tinnitus}

Tinnitus can be regarded as a perceptual correlate of altered spontaneous electrical activity in the central auditory pathway. Previous studies have shown that application of salicylate changes the neuronal spontaneous activity in several parts of the auditory pathway. Salicylate induced elevated central auditory activity has been interpreted as a correlate of tinnitus. Basta and Ernst (2004) studied the direct effect of salicylate application on the spontaneous activity of the mouse inferior colliculus neurons in brain 
slices. They found that salicylate application induced a general increase of electrophysiological activity in IC neurons, and suggested that the inferior colliculus could play an important role in the salicylate-induced generation of tinnitus. However, in Ma et al.'s study (2006) salicylate suppressed the spontaneous activity in the ICC of salicylate-treated mice. Therefore, salicylate-induced changes in spontaneous activity remain controversial and differ considerably between studies. In the present study reduction in the threshold of firing of ICC neurons following salicylate application suggests that salicylate increases membrane excitability of ICC neurons, which may account for the increase in their spontaneous activities. However, whether salicylate affects the spontaneous neurotransmitter release or activity of synaptic receptors in ICC neurons is not clear. Further study is required to answer these questions, and achieve a better understanding of how salicylate changes the spontaneous activity in the ICC.

The analysis of effects of salicylate on intrinsic properties of ICC neurons did not show any change in passive membrane properties or firing frequency of the neurons at levels of below $200 \mathrm{pA}$ of depolarizing current injection. There was, however, a decrease in firing frequency at current levels of 200 to $400 \mathrm{pA}$. It has been estimated that the intensity level of acoustic stimuli normally heard by animals would result in a depolarization of 5-15 mV in a neuron (Kuwada et al., 1997). Therefore, the higher levels of current pulse (200 - $400 \mathrm{pA})$ used in the present study may correspond to much higher sound intensity levels than physiological audible levels. Therefore, it seems that salicylate does not substantially affect signal processing at the physiological range of hearing. 
Further study is needed to investigate effects of salicylate on each type of ion channels separately by application of specific voltage-gated ion channel blockers. Both voltage and current patch clamp recording techniques should be used. Through this approach, it would be possible to determine exact effects of salicylate on different types of ion channels and to relate these changes to those observed in the present study. Since tinnitus is considered to be a consequence of imbalance between excitation and inhibition in central auditory pathways, synaptic studies in brain slice preparations of ICC neurons after salicylate application would be the next step to determine how salicylate affects excitatory or inhibitory transmission. It has been shown that salicylate reduced inhibitory postsynaptic currents in neurons of rat auditory cortex, which may contribute to the elevated excitability of the auditory cortex neurons in animal models of tinnitus (Wang et al., 2006). Their data suggest that suppression of inhibitory synaptic transmissions in the central auditory system can be one of the cellular mechanisms for tinnitus caused by salicylate. In addition, a recent in vivo study on effects of salicylate on the expressions of GABA and glutamate in the IC neurons with immunohistochemical methods has shown that administration of sodium salicylate increased the expression of glutamate-positive neurons and reduced the expression of GABA-positive neurons in the inferior colliculus (Yin et al., 2006). Therefore, a future in vitro electrophysiological study should investigate effects of salicylate on both GABAergic and glutamatergic neurotransmission in ICC neurons. 


\section{Conclusion}

The effects of salicylate on intrinsic properties ICC neurons were examined using a whole-cell patch clamp technique in current clamp mode. The results indicated that salicylate did not have any effect on the passive membrane properties of the neurons, including resting membrane potential, input resistance and time constant. Salicylate also did not show any effect on AP properties except for reduction of AHP amplitude. However, salicylate had prominent effects on firing abilities of the ICC neurons, including the current threshold and firing frequency. It decreased the threshold current required to evoke an action potential. In addition, there was a decrease in firing frequency of the ICC neurons at levels of 200 to $400 \mathrm{pA}$ of depolarizing current injection during salicylate application in comparison to normal control conditions. The APs gradually became smaller and longer with increases in the level of depolarizing current injections, so that at higher levels of depolarizing current only a few spikes followed by several small humps were elicited during salicylate. Salicylate also reduced the AHP amplitude, but did not change the AHP half-width, depolarizing sag during hyperpolarization and rebound after hyperpolarization.

The present study was the first of its kind to examine the effects of salicylate on physiological properties of ICC neurons using whole-cell patch clamp recordings. This approach allowed us to analyze changes in intrinsic properties of ICC neurons following salicylate application, and to examine the possible correlation of these changes with neural mechanisms of tinnitus induced by salicylate. 


\section{References}

Ahuja TK, Wu SH. Intrinsic membrane properties and synaptic response characteristics of neurons in the rat's external cortex of the inferior colliculus. Neuroscience. 2007; 145 (3): 851-65.

Aitkin L. The Auditory Midbrain. Structure and function in the central auditory pathway. Humana press, Clifton, 1986.

Basta D, Ernst A. Effects of salicylate on spontaneous activity in inferior colliculus brain slices. Neurosci Res. 2004; 50: 237-243.

Bauer CA, Brozoski TJ, Holder TM, Caspary DM. Effects of chronic salicylate on GABAergic activity in rat inferior colliculus. Hear Res. 2000; 147: 175-182.

Boettcher FA, Salvi RJ. Salicyalte Ototoxicity: Review and Synthesis. Am J Otolarygnol. $1991 ; 12: 33-47$.

Brown RD, Penny JE, Henley CM, Hodges KB, Kupetz SA,Glenn DW, Jobe JC. Ototoxic drugs and noise. Ciba Found Symp. 1981; 85: 151-171.

Brummett RE. A mechanism for tinnitus? In: Vernon J, Moller A (Eds.), Mechanisms of tinnitus. Allyn and Bacon, MA, 1995, pp. 7-10.

Cazals Y. Auditory sensori-neural alterations induced by salicylate. Prog Neurobiol. $2000 ; 62: 583-631$.

Chen GD, Jastreboff PJ. Salicylate-induced abnormal activity in the inferior colliculus of rats. Hear Res. 1995; 82: 158-178.

Cransac H, Peyrin L, Cottet-Emard JM, Farhat F, Pequignot JM, Reber A. Aging effects on monoamines in rat medial vestibular and cochlear nuclei. Hear Res. 1996; 100: $150-156$.

Day RO, Graham GG, Bieri D, Brown M, Cairns D, Harris G, Hounsell J, PlattHepworth S, Reeve R, Sambrook PN, Smith J. Concentration-response relationships for salicylate-induced ototoxicity in normal volunteers. Br J Clin Pharm. 1989; 28: 695-702.

Didier A, Miller JM, Nuttall AL. The vascular component of sodium salicylate ototoxicity in the guinea pig. Hear Res. 1993; 69: 199-206.

Dobie RA. Depression and tinnitus. Otolaryngol Clin North Am. 2003; 36: 383-388.

Duan M, Agerman K, Ernfors P, Canlon B. Complementary roles of neurotrophin 3 and a $\mathrm{N}$-methyl-D aspartate antagonist in the protection of noise and aminoglycoside- 
induced ototoxicity. Proc Natl Acad Sci USA. 2000; 97: 7597-7602.

Eggermont JJ. On the pathophysiology of tinnitus: a review and a peripheral model. Hear Res. 1990; 48: 111-123.

Eggermont JJ. Central tinnitus. Auris, Nasus, Larynx. 2003; 30 Suppl: S7-12.

Eggermont JJ, Kenmochi M. Salicylate and quinine selectively enhance spontaneous firing rates in secondary auditory cortex. Hear Res. 1998; 117: 149-160.

Eggermont JJ, Roberts LE. The neuroscience of tinnitus. Trends Neurosci. 2004; 27(11): 676-682.

Ehret G. The auditory midbrain, a shunting-yard of acoustical information processing. In: Ehret G and Romand R (Eds.), The central auditory system. Oxford University Press, New York, 1997, pp. 259-316.

Evans EF, Borewe TA. Ototoxic effects of salicylate on the responses of single cochlear nerve fibers and on cochlear potentials. Brit J Audiol. 1982; 16: 101-108.

Evans EF, Wilson JP, Borerwe TA. Animal models of tinnitus. Ciba Found Symp. 1981; 85: $108-138$.

Faye-Lund H, Osen KK. Anatomy of the inferior colliculus in rat. Anat Embryol (Berl). 1985; 171: 1-20.

Guitton MJ, Caston J, Ruel J, Johnson RPR, Puel J. Salicylate induces tinnitus through activation of cochlear NMDA receptors. J Neurosci. 2003; 23(9): 3944-3952.

Heller AJ. Classification and epidemiology of tinnitus. Otolaryngol Clin North Am. 2003; 36: 239-248.

House JW, Brackman DE. Tinnitus: surgical treatment. In: Evered D, Lawrenson G (Eds.), Tinnitus. CIBA Found Symposium. 1981; 85: 204-16.

Houser CR, Esclapez M. Vulnerability and plasticity of the GABA system in the pilocarpine model of spontaneous recurrent seizures. Epilepsy Res. 1996; 26 (1): 207-218.

Jastreboff PJ. Phantom auditory perception (tinnitus): mechanisms of generation and perception. Neurosci Res. 1990; 8: 221-254.

Jastreboff PJ, Hansen R, Sasaki PG, Sasaki CT. Differential uptake of salicylate in serum, cerebrospinal fluid, and perilymph. Arch Otolaryngol Head Neck Surg. 1986; 112: 1050-1053. 
Jastreboff PJ, Sasaki CT. Salicylate-induced changes in spontaneous activity of single units in the inferior colliculus of the guinea pig. J Acoust Soc Am. 1986; 80: 1384-1391.

Jastreboff PJ, Sasaki CT. An animal model of tinnitus: a decade of Development. Am J Otology. 1994; 15: 19-27.

Jenkins WM, Masterton RB. Sound localization: effects of unilateral lesions in central auditory system. J Neurophysiol. 1982; 47: 987-1016.

Jensen K, Jensen MS, Lambert JD. Role of presynaptic L-type $\mathrm{Ca}^{2+}$ channels in GABAergic synaptic transmission in cultured hippocampal neurons. J Neurophysiol. 1999; 81: 1225-1230.

Jensen K, Mody I. L-type $\mathrm{Ca}^{2+}$ channel-mediated short-term plasticity of GABAergic synapses. Nat Neurosci. 2001; 4: 975-976.

Kaltenbach JA, Godfrey Da, Neumann JB, McCaslin DL, Afman CE, Zhang J. Changes in spontaneous neural activity in the dorsal cochlear nucleus following exposure to intense sound: relation to threshold shift. Hear Res. 1998; 124: 78-84.

Kaltenbach JA, Zhang J, Afman CE. Plasticity of spontaneous neural activity in the dorsal cochlear nucleus after intense sound exposure. Hear Res. 2000; 147: 282292.

Kaltenbach JA, Rachel JD, Mathog TA, Zhang J, Falzarano PR, Lewandowski M. Cisplatin-induced hyperactivity in the dorsal cochlear nucleus and its relation to outer hair cell loss: relevance to tinnitus. J Neurophysiol. 2002; 88: 699-714.

Kanold PO, Manis PB. Transient potassium currents regulate the discharge patterns of dorsal cochlear nucleus pyramidal cells. J Neurosci. 1999; 19(6): 2195-208.

Kaufman DL, Houser CR, Tobin AJ. Two forms of gamma- aminobutyric acid synthetic enzyme glutamate decarboxylase have distinct intraneuronal distributions and cofactor interactions. J Neurochem. 1991; 56: 7203-7230.

Kenmochi M, Eggermont JJ. Salicylate and quinine affect the central nervous system. Hear Res. 1997; 113: 110-116.

Koch U, Grothe B. GABAergic and glycinergic inhibition sharpens tuning for frequency modulations in the inferior colliculus of the big brown bat. J Neurophysiol. 1998; 80(1): 71-82.

Koch U, Grothe B. Interdependence of spatial and temporal coding in the auditory midbrain. J Neurophysiol. 2000; 83(4): 2300-14. 
Koch U, Grothe B. Hyperpolarization-activated current (Ih) in the inferior colliculus: distribution and contribution to temporal processing. J Neurophysiol. 2003; 90(6): 3679-87.

Koester J, Siegelbaum SA. Propagated signalling: The action potential. In: Kandel ER, Schwartz JH, Jessell TM (Eds.), Principles of neural science. McGraw-Hill, New York, 2000, pp. 158.

Kuwada S, Batra R, Yin TC, Oliver DL, Haberly LB, Stanford TR. Intracellular recordings in response to monaural and binaural stimulation of neurons in the inferior colliculus of the cat. J Neurosci. 1997; 17(19): 7565-81.

Levine RA, Abel M, Cheng H. CNS somatosensory-auditory interactions elicit or modulate tinnitus. Exp Brain Res. 2003; 153: 643-648.

Li Y, Evans MS, and Faingold CL. In vitro electrophysiology of neurons in subnuclei of rat inferior colliculus. Hear Res. 1998; 121: 1-10.

Liang Y, Zhong NC. Effects of sodium salicylate and extracellular $\mathrm{K}^{+}$on outward potassium current and resting potential of outer hair cell isolated from guinea pig cochlea. Di Yi Jun Yi Da Xue Xue Bao. 2002; 22: 137-140.

Liberman MC, Kiang NYS. Acoustic trauma in cats. Cochlear pathology and auditorynerve activity. Act Otolaryngol Suppl. 1978; 358: 1-63.

Liu J, Li X, Wang L, Dong Y, Han H, Liu G. Effects of salicylate on serotoninergic activities in rat inferior colliculus and auditory cortex. Hear Res. 2003; 175: 4553.

Liu Y, Li X. Effects of salicylate on voltage-gated sodium channels in rat inferior colliculus neurons. Hear Res. 2004; 193: 68-74.

Liu Y, Li X. Effects of salicylate on transient outward and delayed rectifier potassium channels in rat inferior colliculus neurons. Neurosci Lett. 2004; $369: 115-120$.

Liu Y, Li X, Ma C , Liu J, Lu H. Salicylate blocks L-type calcium channels in rat inferior colliculus neurons. Hear Res. 2005; 205: 271-276.

Lockwood AH, Wack DS, Burkard RF, Coad ML, Reyes SA, Arnold SA, Savi RJ. The functional anatomy of gaze-evoked tinnitus and sustained lateral gaze. Neurol. 2001; 56: 472-480.

Lue AJ, Brownell WE. Salicylate induced changes in outer hair cell lateral wall stiffness. Hear Res. 1999; 135: 163-168. 
Lukas-Rüttiger, Jürgen C, Hans-Peter Z, Marlies K. A behavioral paradigm to judge acute sodium salicylate-induced sound experience in rats: a new approach for an animal model on tinnitus. Hear Res. 2003; 180: 39-50.

Ma W, Hidaka H, May BJ. Spontaneous activity in the inferior colliculus of CBA/J mice after manipulations that induce tinnitus. Hear Res. 2006; 212: 9-21.

Manabe Y, Yoshida S, Saito H, Oka H. Effects of lidocaine on salicylate-induced discharge of neurons in the inferior colliculus of the guinea pig. Hear Res. 1997; 103: $192-198$.

Marrion NV, Tavalin SJ. Selective activation of $\mathrm{Ca}^{2+}$-activated $\mathrm{K}^{+}$channels by colocalized $\mathrm{Ca}^{2+}$ channels in hippocampal neurons. Nature. 1998; 395: 900-905.

Melcher JR, Sigalovsky IS, Guinan JJ JR, Levine RA. Lateralized tinnitus studied with functional magnetic resonance imaging: abnormal inferior colliculus activation. J Neurophysiol. 2000; 83: 1058-1072.

Milbrandt JC, Holder TM, Wilson MC, Salvi RJ, Caspary DM. GAD levels and muscimol binding in rat inferior colliculus following acoustic trauma. Hear Res. 2000; 147: 251-260.

Mulheran M. The effects of quinine on cochlear nerve fibre activity in the guinea pig. Hear Res. 1999; 134: 145-152.

Müller M, Klinke R, Arnold W, Oestreicher E. Auditory nerve fibre responses to salicylate revisited. Hear Res. 2003; 183: 37- 43.

Murakami N, Ishibashi H, Katsurabayashi S, Akaike N. Calcium channel subtypes on single GABAergic presynaptic terminal projecting to rat hippocampal neurons. Brain Res. 2002; 951: 121-129.

Noreña AJ, Eggermont JJ. Changes in spontaneous neural activity immediately after an acoustic trauma: implications for neural correlates of tinnitus. Hear Res. 2003; 183: $137-153$.

Ochi K, Eggermont JJ. Effects of salicylate on neural activity in cat primary auditory cortex. Hear Res. 1996; 95: 63-76.

Ochi K, Eggermont JJ. Effects of quinine on neural activity in cat primary auditory cortex. Hear Res. 1997; 105: 105-118.

Oliver DL. Neuronal organization in the inferior colliculus. In: Winer JA and Schreiner CE (Eds.), The Inferior Colliculus. Springer-Verlag, New York, 2005, pp. 69-114. 
Peng B, Chen S, Lin X. Aspirin selectively augmented N-methyl-D-aspartate types of glutamateresponses in cultured spiral ganglion neurons of mice. Neurosci Lett. $2003 ; 343: 21-24$.

Peruzzi O, Sivaramakrishnan S, Oliver DL. Identification of cell types in brain slices of the inferior colliculus. Neurosci. 2000; 101(2): $403-16$.

Rouiller EM. Functional organization of the auditory pathways. In: Ehret G and Romand R (Eds.), The central auditory system. Oxford University Press, New York, 1997, pp. 3-65.

Russier M, Carlier E, Ankri N, Fronzaroli L, Debanne D. A-, T-, and H-type currents shape intrinsic firing of developing rat abducens motoneurons. J Physiol. 2003; 549: 21-36.

Sachanska T. Changes in blood serotonin in patients with tinnitus and other vestibular disturbances. Int Tinnitus J. 1999; 5 (1): 20-32.

Salvi RJ, Wang J, Ding D. Auditory plasticity and hyperactivity following cochlear damage. Hear Res. 2000; 147: 261-274.

Seki S, Eggermont JJ. Changes in spontaneous firing rate and neural synchrony in cat primary auditory cortex after localized tone-induced hearing loss. Hear Res. 2003; 180: $28-38$.

Shehata WE, Brownell WE, Dieler R. Effects of salicylate on shape, electromotility and membrane characteristics of isolated outer hair cells from guinea pig cochlea. Acta Otolaryngol. 1991; 111: 707-718.

Sivaramakrishnan S, Oliver DL. Distinct K currents result in physiologically distinct cell types in the inferior colliculus of the rat. J Neurosci. 2001; 21: 2861- 2877.

Sivaramakrishnan S, Oliver DL. The generation of rebound excitation in the inferior colliculus. Assoc. Res. Otolaryngol. 2006; 29: 51-52.

Smith PH. Anatomy and physiology of multipolar cells in the rat inferior collicular cortex using the in vitro brain slice technique. J Neurosci. 1992; 12(9): 3700-15.

Stypulkowski PH. Mechanisms of Salicylate Ototoxicity. Hear Res. 1990; 46: 113-145.

Wallhausser-Franke E. Salicylate evokes c-fos expression in the brain stem: Implications for tinnitus. NeuroRep. 1997; 8: 725-728.

Wallhausser-Franke E, Braun S, Langner G. Salicylate alters 2-DG uptake in the auditory system: a model for tinnitus? NeuroRep. 1996; 7: 1585-1588. 
Wallhausser-Franke E, Mahlke C, Oliva R, Braun S, Wenz G, Langner G. Expression of c-fos in auditory and non-auditory brain regions of the gerbil after manipulations that induce tinnitus. Exp Brain Res. 2003; 153 (4): 649-654.

Wang HT, Luo B, Zhou KQ, Xu TL, Chen L. Sodium salicylate reduces inhibitory postsynaptic currents in neurons of rat auditory cortex. Hear Res. 2006; 215 (1-2): 77-83.

Willott JF, Aitkin LM, McFadden SL. Plasticity of auditory cortex associated with sensorineural hearing loss in adult C57BL/6J mice. J Comp Neurol. 1993; 329: $402-411$.

Winer JA, Schreiner CE. The central auditory system: A functional analysis. In: Winer JA and Schreiner CE (Eds.), The Inferior Colliculus. Springer Verlag, New York, 2005, pp. 1-68.

$\mathrm{Wu}$ SH. Biophysical properties of inferior colliculus Neurons. In: Winer JA and Schreiner CE (Eds.), The inferior colliculus. Springer-Verlag, New York, 2005, pp. 282-311.

$\mathrm{Wu}$ SH, Ma CL, Sivaramakrishnan S, and Oliver DL. Synaptic modification in neurons of the central nucleus of the inferior colliculus. Hear Res. 2002; 168(1-2): 43-54.

Yin SH, Tang AZ, Xing XL, Tan SH, Xie LH. Effects of sodium salicylate on the expressions of gamma-aminobutyricacid and glutamate and auditory response properties of the inferior colliculus neurons. Sheng Li Xue Bao. 2006; 58(5): 44955.

Zenner HP, Ernst A. Cochlear-motor, transduction and signal- transfer tinnitus: models for three types of cochlear tinnitus. Eur Arch Otorhinolaryngol. 1993; 249: 447454.

Zhang JS, Kaltenbach JA, Wanga J, Bronchti G. Changes in [14C]-2-deoxyglucose uptake in the auditory pathway of hamsters previously exposed to intense sound. Hear Res. 2003; 185: 13-21. 